\title{
On memory, dimension, and atmospheric teleconnections
}

DOI 10.1515/mcwf-2017-0001

Received October 11, 2016; accepted February 10, 2017

\begin{abstract}
Using reanalysed atmospheric data and applying a data-driven multiscale approximation to nonstationary dynamical processes, we undertake a systematic examination of the role of memory and dimensionality in defining the quasi-stationary states of the troposphere over the recent decades. We focus on the role of teleconnections characterised by either zonally-oriented wave trains or meridional dipolar structures. We consider the impact of various strategies for dimension reduction based on principal component analysis, diagonalization and truncation. We include the impact of memory by consideration of Bernoulli, Markovian and non-Markovian processes. We a priori explicitly separate barotropic and baroclinic processes and then implement a comprehensive sensitivity analysis to the number and type of retained modes. Our results show the importance of explicitly mitigating the deleterious impacts of signal degradation through ill-conditioning and under sampling in preference to simple strategies based on thresholds in terms of explained variance. In both hemispheres, the results obtained for the dominant tropospheric modes depend critically on the extent to which the higher order modes are retained, the number of free model parameters to be fitted, and whether memory effects are taken into account. Our study identifies the primary role of the circumglobal teleconnection pattern in both hemispheres for Bernoulli and Markov processes, and the transient nature and zonal structure of the Southern Hemisphere patterns in relation to their Northern Hemisphere counterparts. For both hemispheres, overfitted models yield structures consistent with the major teleconnection modes (NAO, PNA and SAM), which give way to zonally oriented wavetrains when either memory effects are ignored or where the dimension is reduced via diagonalising. Where baroclinic processes are emphasised, circumpolar wavetrains are manifest.
\end{abstract}

Keywords: atmospheric teleconnections, stochastic modelling, dimension reduction

\section{Introduction}

In their seminal paper, Lau et al. [25] describe the low frequency variability of the major atmospheric circulation modes in terms of multi-scale interactions. In addition to examining the low frequency variability of the major zonal and meridional modes of variability in the tropics and higher latitudes, they point to the need for a unified framework for the manipulation and analysis of observational data. Crucially, they caution against approaches reliant on prefiltering of the data and specifically note that: "prefiltering procedures are legitimate only if the features in question occur with distinct timescales that fall within the chosen frequency window”, which for atmospheric phenomena occurring on a continuum of scales $[26,45]$ is not often the case. As

\footnotetext{
^Corresponding Author: Terence. J. O’Kane: Climate Science Centre, CSIRO Oceans and Atmosphere, Hobart, Australia Didier P. Monselesan: Climate Science Centre, CSIRO Oceans and Atmosphere, Hobart, Australia James S. Risbey: Climate Science Centre, CSIRO Oceans and Atmosphere, Hobart, Australia Illia Horenko: Universita della Svizzera, Italiana, Lugano, Switzerland Christian L. E. Franzke: Meteorological Institute and Center for Earth System Research and Sustainability, University of Hamburg, Hamburg, Germany
} 
an example, the Southern Annular Mode (SAM) [44], typically characterised as the large scale zonal component of the midlatitude tropospheric flow, presents as the leading mode of variability for geopotential height in principal component (PC) - empirical orthogonal function (EOF) analysis. However, such an approach, based on maximising the variance for a particular invariant spatial pattern, gives the misleading impression that the zonal flow occurs only at the large scales, when in fact the SAM is multiscale with a wave- 3 pattern embedded in the zonal flow. PC analysis is a standard tool in atmospheric science to reduce the data to a few leading modes of variability. While a useful tool for dimension reduction, it is important to bear in mind that, whereas the leading PC/EOF modes may often have readily interpretable physical behaviours, often the higher order modes do not correspond by themselves to observable coherent features, rather a combination of modes can be required to characterise a physical dynamical mode, e.g. blocking.

When prefiltering has been performed, i.e. averaging, intrinsic variability on timescales shorter than the averaging period is removed and in addition causal connections are affected. More generally, analysis based solely on methods where the dimensionality is reduced, either by PC or EOF analysis, such that only the leading few modes are retained, and often justified in terms of projection onto an arbitrarily determined slow manifold [27, 29], can also be interpreted as a form of prefiltering. Where scale separation is absent, arbitrary filtering (averaging) and truncation of the underlying data, common to many studies of low frequency climate variability [9], can lead to the misrepresentation of the relative importance of the drivers of variability at given spatio-temporal scales [36]. In general, more sophisticated stochastic approaches to mode reduction must be applied and, in particular, where some form of parameterization of the unresolved scales is incorporated $[7,10,11,13,30,31]$.

Determining the parameters of the optimal stochastic model in the relatively short timeseries of the available atmospheric reanalysis data represents a challenge. To illustrate why, consider a general stationary Vector Auto-Regressive (VAR) stochastic model of the form:

$$
\mathbf{x}_{t}=\mu+\sum_{i=1}^{M E M} \mathbf{A}_{i} \mathbf{x}_{t-i \tau}+\epsilon_{t}
$$

where $\mathbf{x}_{t}$ is the data, $\mu$ is the mean, $\mathbf{A}_{i}$ is the set of model parameters to be determined for a given time lag $i \tau$ up to a maximum lag $M E M$ and $\epsilon_{t}$ are independent identically distributed (i.i.d) random terms. In such models it is easily shown that the total number of free model parameters, even in the most restrictive case of a Bernoulli model (random without memory effects), can still be of the order of many thousands. Since the length of even the longest available observational (reanalysed) atmospheric data is limited to a few decades at daily resolution (of order $2 \times 10^{4}$ degrees of freedom), a classical ill-posed problem manifests in the overfitting of the data. Ill-conditioning occurs when the number of degrees of freedom in the in the data is not much greater than the number of free parameters in the model to be fitted. The ill-conditioning will be even further exacerbated when considering non-stationary data sets with secular trends. That said, discerning the most informative and well-posed model from the many possible choices can be successfully achieved via application of information theoretic approaches for certain classes of stochastic models, such as the Bayesian (BIC) or Akaike (AIC) information criteria for multi-linear regression for example, that implicitly penalise for ill-conditioning by attributing much higher values to the least well posed models [8, 32]. For non-stationary and multiscale systems without clear spatio-temporal scale separation (most atmospheric problems), mode reduction by stochastic methods requires the parameterization of the unresolved scales. In the particular case of VAR methods, it is sufficient to show that the residuals have a (log-)normal distribution to justify parameterization of the unresolved scales by an i.i.d stochastic term.

For many atmospheric phenomena, low frequency variability manifests as transitions between quasistationary states or regimes $[6,26]$. Transitions between regimes are often initiated by weak stochastic forcing of the large scale slow modes by the fast noisy small scales [5, 34], or through the organised amplification of initially small disturbances via the inverse energy cascade such as occurs in quasi-two dimensional geophysical flows [24]. In multi-scale systems that undergo regime transitions, for example atmospheric blocking [6], it is critical that the unresolved scales, or indeed discarded dimensions, must be modelled or parameterised in some way, i.e. time dependence of the model parameters can be induced by the influence of the unresolved scales leading to large scale regime transitions between quasi-stationary states [38]. In the troposphere, these 
quasi-stationary states are associated with the major teleconnections or persistent height anomalies (blocking). In statistical mechanics and dynamics, both stochastic [22, 48] and deterministic [35] approaches exist to parameterize the drain and injection of energy/enstrophy from the retained to the subgrid scales - processes respectively known as eddy viscosity and stochastic backscatter.

Recently in a series of papers $[8,37,38,41]$, the dynamics of tropospheric persistent states and their secular trends have been examined using data-driven multiscale methods that allow for approximation to nonstationary dynamical processes [13-17] including parameterization of subgrid scales by stochastic forcing. This work, applying the finite element, bounded variation, vector autoregressive method (FEM-BV-VARX), highlighted the pivotal role of the jet stream (polar and subtropical jets) acting as a waveguide to provide meridional trapping of a zonally elongated wave patterns in both the Southern (SH) [38] and Northern (NH) [41] hemispheres.

Risbey et al. [41] also applied FEM-BV-VARX to assess NH mid-tropospheric flow regimes characterised by regime switches between metastable states. They assessed 500hPa geopotential height anomalies from three different reanalysis sets confirming the utility of surface only reanalyses for capturing mid-tropospheric variability. In addition to the $\mathrm{NH}$, they examined a set of regional domains yielding structures consistent with the major NH meridional: (Pacific North American (PNA) [47] and North Atlantic Oscillation (NAO) [20, 47]) and zonal (Scandinavian blocking over Eurasia) teleconnection modes. In particular, they found the NH states comprised aspects of an annular mode (AO) $[28,43,44]$ and a Circumglobal teleconnection Wave 5 Pattern (CWP) in the midlatitudes consistent with that described previously by Branstator [4]. They further found that some of the nodes in CWP manifest as part of regional meridional dipole structures of the PNA and NAO. This contrasts with the Southern Hemisphere [38], where the circumglobal waveguide exhibits wave number 5-6 with little or no tendency for persistent dipolar structures but rather regional manifestations of the zonal wavetrain associated with blocking and the Pacific South American (PSA) pattern [25, 33]. Importantly, Risbey et al. [41] showed that not only are the FEM-BV-VARX NH composite patterns consistent with teleconnections derived empirically (often as leading EOF modes), but that the model affiliations from FEM-BV-VARX are highly correlated with standard teleconnection indices.

O'Kane et al. [37] applied the FEM-BV-VARX to the NCEP v1 reanalysis data set (1948 to 2009) [21] in order to characterise changes in the Southern Hemisphere 500-hPa circulation. They found the SH circulation underwent a regime transition from one dominated by switching between positive and negative phases of the planetary wave 3 pattern to one post-1978 characterised by a strong secular trend toward the positive phase of the SAM at the expense of blocking. Franzke et al. [8] subsequently applied the FEM-BV-VARX to distinguish between natural variability and the response to external (radiative) forcing thereby providing observational evidence that anthropogenic greenhouse gas concentrations have been the major driver of the secular trends in the SAM and blocking over recent decades. This work supports the premise that the recovery of the ozone hole might not be effective in largely mitigating the poleward shift of the midlatitde jet in response to global warming [39].

In a subsequent study of the high resolution JRA-55 reanalysis [23], O'Kane et al. [38] identified the dynamical drivers of systematic changes in persistent quasi-stationary states (regimes) of the polar and subtropical jets of the Southern Hemisphere troposphere and their secular trends. Using a comprehensive set of diagnostics on both isentropic and isobaric surfaces, they showed FEM-BV-VARX composite patterns consistent with a circumglobal wave 3 (polar)-wave 5 (subtropical) pattern, while regional composites reveal the PSA pattern and blocking modes. The respective roles of potential vorticity sources, stationary Rossby waves and baroclinic instability on the dynamics of these circulation modes were shown to be reflected by the seasonal variations of the waveguides, where Rossby wave sources and baroclinic disturbances are largely contained within the waveguides with little direct evidence of sustained remote tropical influences on persistent synoptic features consistent with Lau et al. [25]. Secular trends were again shown to be driven by changes in $\mathrm{CO}_{2}$ concentration. Horenko et al. [18] further advanced the methodology in order to extract the statistically most significant Bayesian causality relations between empirical indicies of the seasonal climate teleconnections time series and thus quantify the probabilistic causality impacts due to the unresolved/weather scales (i.e. beyond and above the synoptic scales) on the major climate teleconnection modes. 
In the aforementioned studies, the time series of model affiliations was obtained via application of FEMBV-VARX to $500 \mathrm{hPa}$ geopotential height anomalies from which composites of all diagnostic variables were obtained. Here, we apply FEM-BV-VARX to examine the full three dimensional (3-D) structure of the troposphere. In doing so, we are presented with a severe computational challenge requiring dimension reduction in order to avoid ill-conditioning. The consequences of various dimension reduction strategies is the focus of this manuscript. We will show that a careful and systematic approach to the problem can in fact lead to a deeper insight into the major atmospheric circulation modes and progress toward a more unified approach to analyzing observational data.

The structure of the paper is as follows. In the section 2, we outline the data, diagnostics, methodology and sequence of sensitivity tests. Specifically, the reanalysis data and diagnostics are described in section 2.1. The FEM-BV-VARX method is briefly described in section 2.2. The sequence of sensitivity experiments is detailed in section 2.3. The results, discussion, summary and conclusions are in sections 3, 4 and 5 respectively.

\section{Method}

\subsection{Data and diagnostics}

\subsubsection{JRA-55 reanalysis}

We analyse daily data from the Japanese 55-year Reanalysis (JRA-55) conducted by the Japan Meteorological Agency (JMA). JRA-55 covers the global radiosonde period from 1958 and is the first to apply four-dimensional variational analysis to this period. Details of the JRA-55 are described by Kobayashi et al. [23] including the observations assimilated, data assimilation system, and forecast model including the basic characteristics of the JRA-55 product. JRA-55 was produced with JMA's operational data assimilation system at TL319 resolution with major improvements including a reduced cold bias in the lower stratosphere and a mitigated dry bias in the Amazon basin.

\subsubsection{Diagnostics}

In order to examine the dynamics of persistent states identified using the FEM-BV-VARX framework we consider geopotential height $Z_{g}(m)$, surface air temperature $S A T\left({ }^{0} \mathrm{C}\right)$ and zonal winds $u\left(\mathrm{~ms}^{-1}\right)$ for daily mean data. Geopotential height has units of meters and is defined as $Z_{g}=g z$, where $g$ is gravity and $z$ the vertical coordinate in the direction normal to the geopotential surface [46]. In order to span the troposphere, we consider geopotential height on the $Z_{g 300 h P a}(m), Z_{g 500 h P a}(m)$, and $Z_{g 700 h P a}(m)$ isobaric pressure surfaces.

As a simple diagnostic for incipient baroclinic instability, we apply the Phillips Criteria ( $P C r)$ [40] in terms of the shear between the horizontal winds $u$ at $300 \mathrm{hPa}$ and $700 \mathrm{hPa}$ as

$$
P C r=\bar{u}^{300}-\bar{u}^{700}-\frac{b_{\kappa} c_{p} \bar{\sigma}}{a \Omega} \frac{\cos \phi}{\sin ^{2} \phi} \geq 0,
$$

where $\bar{\sigma}$ is the static stability for a given basic (climatological) state, typically calculated as $1 / 2\left(\theta^{300}-\theta^{700}\right)$ where $\theta$ is the potential temperature at $300 \mathrm{hPa}$ and $700 \mathrm{hPa} . c_{p}=1004 \mathrm{~J} \mathrm{deg}^{-1} \mathrm{~kg}^{-1}$, is the specific heat of air at constant pressure, $\Omega=7.292 \times 10^{-5} \mathrm{rad} \mathrm{s}^{-1}$, is the earth's angular speed of rotation, $b_{\kappa}=0.124$ is a dimensionless constant, $a=6.371 \times 10^{6} \mathrm{~m}$, is the radius of the earth and $\phi$ is latitude. Positive values are indicative that the flow is unstable to small perturbations, i.e. that the mid-tropospheric atmosphere is unstable to storm development. In addition, we will consider the anomalous $P C r$ w.r.t. its daily climatological values. $P C r$ is in units of $\mathrm{ms}^{-1}$. 


\subsection{FEM-BV-VARX method}

The Finite Element Bounded Variation Vector Auto-Regressive with eXternal factors (FEM-BV-VARX) method as applied to atmospheric reanalyses has been described in detail elsewhere $[8,37,38,41]$ and is now well documented $[13,32]$. Here, we provide only a very brief outline of the method and refer interested readers to the citations provided. We apply the FEM-BV-VARX analysis to daily observational (reanalysis) geopotential height anomalies - $Z_{g}^{\prime}$ at 300,500 and $700 \mathrm{hPa}$ - calculated as deviations from the climatological mean but not detrended. The impact of reducing the dimensionality of the $Z_{g}^{\prime}$ data using principal component analysis (PCA) is a primary motivation of this study (see section 2.3). To this end a series of experiments (described in section 2.3) have been undertaken to ascertain the sensitivity to retained dimensions and to how the covariances are constructed.

The approach taken here is to fit a non-stationary stochastic model to the data and determine the optimal set of time evolving free model parameters. Let $\mathbf{x}_{0}, \ldots, \mathbf{x}_{T} \in \Psi \subset \mathbf{R}^{n}$ be the observed $n$-dimensional time series with $T+1$ daily averages in the interval $[0, T]$. Assuming that $\mathbf{x}_{t}$ can be approximated by the timediscrete output of a particular direct mathematical model $F\left[\mathbf{x}_{t}, \ldots, \mathbf{x}_{t-m \tau}, \theta(\mathbf{t}), \mathbf{t}\right]=\mathbf{0}$ where $F(\cdot)$ is the model operator, $t$ is the model time step, $m \tau$ is the memory depth, and $\theta(t):[0, T] \rightarrow \Omega \subset R^{d}$ is a (time-dependent) set of the model parameters with $d$ the dimension of a model parameter space. Following the approach taken by Horenko [15] we next define a model distance functional $g\left[\mathbf{x}_{t}, \theta(t)\right]: \Psi \times \Omega \rightarrow[0, \infty)$ describing the distance between some given $\mathbf{x}_{t}$ at time $t$ and the output of the model $F(\cdot)$ calculated for a fixed set of parameters $\theta(t)$. For a given observation series $\mathbf{x}_{0}, \ldots, \mathbf{x}_{T}$ and some fixed functional form $g(\cdot)$, the inverse problem (or the parameter identification problem) can be approached via the solution of the following variational problem:

$$
\sum_{t=1}^{T} g\left[\mathbf{x}_{t}, \theta(t)\right] \rightarrow \min \theta(t)
$$

subjected to the constraints given above.

The problem as it stands is ill posed and so requires some assumptions to be made about the temporal dependence of the unknown parameters $\theta(t)$. Following $[15,16]$, we now assume that for any $t \in[0, T]$ model distance, functional $g\left[\mathbf{x}_{t}, \theta(t)\right]$ can be represented as a convex linear combination of $K \geq 1$ stationary model distance functionals i.e. model functionals, dependent on some constant (time-independent) model parameters $\theta_{i} \in \Omega, i=1, \ldots, K$ such that

$$
g\left[\mathbf{x}_{t}, \theta(t)\right]=\sum_{i=1}^{K} \gamma_{i}(t) g\left(\mathbf{x}_{t}, \theta_{i}\right)
$$

with some convex set of time-dependent model affiliations $\gamma_{i}(t)$, i.e.

$$
\begin{array}{r}
\sum_{i=1}^{K} \gamma_{i}(t)=1, \quad \forall t \in[0, T], \\
\gamma_{i}(t) \geq 0, \quad \forall t \in[0, T], \quad i=1, \ldots, K
\end{array}
$$

and where we define $\Theta=\left(\theta_{1}, \ldots, \theta_{K}\right)$. The assumption here is that at any time $t$ the global time-dependent (or nonstationary) model distance functional $g\left[\mathbf{x}_{t}, \theta(t)\right]: \Psi \times \Omega \rightarrow[0, \infty)$ can be approximated by one of $K$ local time-independent (or stationary) model distance functionals chosen according to some time-dependent probabilities (or model affiliations) $\Gamma(t)=\left[\gamma_{1}(t), \ldots, \gamma_{K}(t)\right]$.

The FEM-BV-VARX approximates dynamical processes by a stochastic model of the form:

$$
\mathbf{x}_{t}=\mu_{t}+\mathbf{A}(t) \phi_{1}\left(\mathbf{x}_{t-\tau}, \ldots, \mathbf{x}_{t-m \tau}\right)+\mathbf{B}(t) \phi_{2}\left(u_{t}\right)+\mathbf{C}(t) \epsilon_{t}
$$

where more specifically $\Theta(t)=(\mu(t), \mathbf{A}(t), \mathbf{B}(t), \mathbf{C}(t))$ is the vector of time dependent model parameters with mean $\mu(t) . \phi_{1}$ is in general a nonlinear function connecting present and past observations $\left(\mathbf{x}_{t-\tau}, \ldots, \mathbf{x}_{t-m \tau}\right)$, but here we take it to be the linear autoregressive factor model. $\phi_{2}\left(u_{t}\right)$ is an external factor function, and $\mathbf{C}(t)$ 
couples the non-parametric, independent and identicallly-distributed (i.i.d.) noise process $\epsilon_{t}$ to the analysed time series (hereby modelling the impact of unresolved subgrid-scale effects). Time dependence of the model parameters $\Theta(t)$ is also induced by the influence of the unresolved scales and leads to regime transitions in many realistic systems. Here we apply the stochastic model in the absence of any external covariates $\left(\mathbf{B}(t) \phi_{2}(u(t)=0)\right.$ i.e. without forcing.

For a given number, $K$, of clusters and fixed maximal time lag, $m$, the method minimises the distance of the model trajectory (of model metric $g$ ) at each time, $t$, to one of $K$ model clusters. The model metric used is the Euclidean norm measuring the model error as the squared distance between $\mathbf{x}_{t}$ and the output of the average model function $g\left(\mathbf{x}_{t}, \theta(t)\right)=\left\|\mathbf{x}_{t}-\mu(t)-\mathbf{A}(t) \phi_{1}\left(\mathbf{x}_{t-\tau}, \ldots, \mathbf{x}_{t-m \tau}\right)-\mathbf{B}(t) \phi_{2}[u(t)]\right\|_{2}^{2}$ (see Horenko [16] for details). We explicitly compare results for time lags corresponding to Bernoulli (random memoryless: lag 0 ), Markovian (dependent only on the prior timestep: lag 1) and non-Markovian (long term memory effects here we consider memory out to 4 days: lags $2-4$ ). The model affiliation sequence

$$
\Gamma(t)=\left(\gamma_{1}(t), \gamma_{2}(t), \ldots, \gamma_{K}(t)\right)
$$

represents the probabilities of residing in each cluster state. The time-dependent vector $\Gamma(t)=$ $\left(\gamma_{1}(t), \ldots, \gamma_{K}(t)\right)$ contains the probabilities for an observation $x_{t}$ at time $t$ to be described/explained by an output of a vector autoregressive external factor model (VARX) with constant (time-independent) model parameters $\theta_{i} . \Gamma(t)$ together with $\Theta=\theta_{1}, \ldots, \theta_{K}$ are jointly obtained from the numerical optimisation given by Eq. 9. The method treats the clustering of non-stationary multidimensional data, $\mathbf{x}_{t} \in \mathbf{R}^{d}$ as a minimisation problem:

$$
L(\Theta, \Gamma)=\sum_{t=0}^{T} \sum_{i=1}^{K} \gamma_{i}(t) g\left(\mathbf{x}_{t}, u_{t}, \theta_{i}\right) \rightarrow \min (\Gamma(t), \Theta)
$$

subject to convexity constraints $\sum_{i=1}^{K} \gamma_{i}(t)=1, \forall t \in[0, T]$ and $\gamma_{i}(t) \geq 0, \forall t \in[0, T], i=1, \ldots, K$.

To select the proper order parameters for the VARX model, the optimal functional for external factors in Eq. 7 and to account for parsimony, we apply information theoretic criteria. Specifically, we apply the Akaike Information Criteria [1] to assess the goodness of fit relative to the number of fitted parameters or external factors used, allowing one to find the model that is least over-fitting while best fitting the analysed data. In practice the lowest Akaike Information Criterion (AIC) value is chosen, where $A I C=-2 \log L_{\max }+2 M$ with $L_{\max }$ the maximum $\log$ likelihood achievable by the model and $M$ the number of free parameters. The optimal model for the number of clusters, $K$, with a given persistency threshold determined in this way is the best fitting model with the fewest free parameters [32]. It should however be appreciated that AIC can only be applied to discriminate between models of a given dimension. Thus the AIC can only determine the optimal choice of model parameters (including penalizing for ill-conditioning and overfitting) for a given class of stochastic model with the same fixed number of PCs (dimensions) but whose retained memory information may vary. It therefore follows that information theoretic methods cannot discriminate between models whose parameters have different dimensions, i.e. different retained number of PCs.

The number of different spatio-temporal regimes-clusters $\mathrm{K}$, the model parameters to be chosen within these regimes, such as memory depth and number of PCs, and the indicator functions $\gamma_{k}(\cdot)$ signalling activation of the respective models, are all determined simultaneously in a global optimisation procedure. This yields a judicious compromise between low residuals in reproducing the data of a training set on the one hand, and the demand for the smallest-possible overall number of free parameters of the complete model on the other. The resulting FEM-BV-framework is essentially parameter free apart from the overall number of optimisation repetitions (annealing steps) with different randomly-chosen initial values $\Gamma$ or $\Theta$ for parameter optimisation. Increasing this number reduces the probability of getting trapped in one of the local minima of $L$ (for $N_{C}>0$ ), simultaneously linearly increasing the amount of computations. Therefore, the number of annealing steps should be chosen carefully, dependent on the available computational resources and the size of the data to be analysed. In an earlier study [38] we considered 4, 8, 16, 32, 64, 128 and 256 annealing steps in order to rigorously test sensitivity to this parameter choice. We show only results for 256 annealing steps and the FEM-BV-VARX conducted using $Z_{g}^{\prime}$ data here. 
A key output of the FEM-BV-VARX method is the posterior model affiliation sequence (or Viterbi path) describing the most likely cluster state, $i$, of the system at each time. From the Viterbi path, one can construct composites by averaging $\mathbf{x}$, here corresponding to geopotential height anomalies, over all times when the system is in each of the respective regime states corresponding to the cluster states $i=1, \ldots, K$. For each of the hemispheres, we determined via the global optimization procedure that $K=2$ cluster states, corresponding to the positive and negative phases of the dominant quasi-stationary teleconnection modes, was optimal. The composites show the spatial structure of each (metastable) cluster state. Although not the focus here, the Viterbi path also allows a construction of the residence behaviour of the system in switching between each cluster state and allows for the identification of secular trends in each of the regime states. Composites for each metastable state were constructed annually and for each of the seasons (DJF, MAM, JJA, SON) and for the diagnostic variables indicated previously.

\subsection{Sensitivity experiments}

The impact of reducing the dimensionality of the $Z_{g}^{\prime}$ data, here using PCA is examined in a series of experiments described in table 1. Previous studies at a given geopotential height have shown that between 9 and 20 retained PCs is sufficient to characterise the persistent teleconnections of the tropospheric circulation $[8,37,38,41]$. Unlike the aforementioned studies, here we consider the 3-D sructure of the troposphere requiring certain choices to be made regarding retained PCs. Retaining a large number of PCs in order to explain a significant amount of the variance will likely lead to a highly ill-conditioned problem. We explore several dimension reduction strategies, prior to application of the FEM-BV-VARX methodology. Specifically, PCA is carried out on geopotential height anomalies $Z_{g}$ at 300, 500, and 700hPa levels. When combining PCs prior to performing the singular value decomposition the anomalies, are weighted by $\sqrt{\cos (\text { lat })}$ and then standardised by the maximum rms over all time. In this way, PCs at various geopotential heights are normalised prior to combining. Subsequent diagonalizing or a substantial reduction of the number of retained PCs are employed to mitigate ill-conditioning when identified and to test sensitivity to dimensionality. These experiments are detailed in table 1.

\subsection{Dimension reduction strategies}

In order to reduce the dimensionality of the data, prior to application of the FEM-BV-VARX methodology, Principal Component Analysis (PCA) is carried out on geopotential height anomalies $Z_{g}$ at 300-500-700hPa levels. Two approaches are taken to elucidate the role of baroclinic and barotropic processes. The first approach, denoted multi-, is the simultaneous multi-level calculation of the leading 60 PCs in order to identify only equivalent barotropic structures. Here (Expt. 1) we first reduce the data by applying singular value decomposition (SVD) in the $z$ direction at each grid point $(x, y)$ location i.e. $\mathbf{X}=(x, y)$ i.e. (lat,lon) and $z=$ vertical levels.

$$
\left(\begin{array}{l}
300 h P a \\
500 h P a \\
700 h P a
\end{array}\right) \rightarrow S V D(z \times \text { time })
$$

in order to identify only structures that have common variance in the vertical direction. We then retain the resulting leading mode at each grid point and reconstruct a reduced data set. Next SVD is applied to the reconstructed data at all levels simultaneously, i.e. $\operatorname{SVD}\left(\mathbf{X}^{\prime} \cdot W\right)$ where $W=\sqrt{\cos (l a t)}$, and $\mathbf{X}^{\prime}$ is the transpose.

For the comb-case (Expt. 5), SVD is first applied to calculate the leading 20 PCs independently at each level after which they are combined. Application of $S V D$ to each level independently, allows for inclusion of baroclinic processes associated with onset and decay of persistent states. It is important to note that in all cases the PCs are normalised prior to application of FEM-BV-VARX. 
Subsequent diagonalizing (Expts. $2 \& 6$ ), or alternately calculation with a substantial reduction in the number of PCs (Expts. 3, 4 \& 7) is then employed to mitigate ill-conditioning and to test sensitivity to dimensionality.

\subsubsection{Diagonalising approximation}

Calculating 60PCs from daily data over the 55 year period post 1958 gives a dimension of 20454(temporal data points) $\times 60(P C s)$. When retaining the dominant $n$ modes the resulting $n$ dimensional VARX model, where each dimension communicates with every other dimension, leads to quadratic growth in the number of VARX parameters in the matrix $A$ (Eqn. 7) and of dimension $n$. As discussed by Franzke et al. [8], this also applies to the stationary VARX model of the general form:

$$
\mathbf{x}_{t}=\mu+\sum_{i=1}^{M E M} \mathbf{A}_{i} \mathbf{x}_{t-i \tau}+\mathbf{B} u_{t}+\epsilon_{t}
$$

(where $\mathbf{x}_{t}$ has a dimension $n, u_{t}$ has a dimension $m$ and $\epsilon_{t}$ is i.i.d.). Where the total number of model parameters $N_{\text {param }}=n+M E M{ }^{\star} n^{2}+n{ }^{\star} m$, with $n=60$ and memory $M E M \geq 2$ the result is in the order of many thousands of free model parameters (see table 1 for values). Given the length of the available observational data considered here, we find ourselves confronted with a classic ill-posed problem manifesting in the potential for over-fitting of the data (for additional discussion see Metzner et al. [32]). While we include terms corresponding to inclusion of external covariates $u(t)$ for completeness, in the calculations that follow in section 3 we will not consider the role of external forcing but focus solely on the role of dimension and memory in the non-stationary problem i.e. $K \geq 2$ where secular trends and multiple metastable states are present. For a detailed examination of the role of external forcing on mid-tropospheric secular behaviour and systematic circulation changes over recent decades, we refer the interested reader to the recent detection and attribution study by Franzke et al. [8].

One approach to ameliorating the problem of over-fitting is to diagonalise the matrix $A_{i}$. Since the offdiagonal elements of $A_{i}$ couple the different dimensions of $x_{t}$, diagonalising is equivalent to a separate identification of the $n$ following problems wrt. $\mu(j), A_{i}(j, j), B(j$, :): i.e.

$$
\forall j=1, n: \quad \mathbf{x}_{t}(j)=\mu(j)+\sum_{i=1}^{M E M} \mathbf{A}_{i}(j, j) \mathbf{x}_{t-i \tau}(j)+\mathbf{B}(j,:) \star u_{t}+\epsilon_{t}
$$

therefore the total number of parameters now grows linearly with $n$ as

$$
N_{\text {param }}=n *(1+M E M+m)
$$

(multiplied by $K$ if considering non-stationary models where $K>1$ ).

Even such a diagonally-restricted VARX-model is however much more general than multilinear regression

$$
\mathbf{x}_{t}=\mu+\mathbf{B} u_{t}+\epsilon_{t}
$$

which is a special case of VARX for $K=1$ and $\mathbf{A}_{j}=0 \forall j$. Thus, even with the diagonality restriction, this analysis is more general than the stationary multilinear regression approaches, e.g. Roscoe and Haigh [42]. As discussed previously, one might further seek to reduce the number of parameters such that the problem is not ill-posed. To this end we consider experiments with a range of retained PCs as described in table 1.

Clearly timeseries analysis where persistency of the respective metastable states is weak represents a serious challenge. One approach we explored assumes that for fixed timeseries $x_{t}$, number of metastable states $K$, and persistency $N_{C}$ and for fixed local VARX parameters $\theta_{i}=\left(\mu^{i}, \mathbf{A}_{1}^{i}, \ldots, \mathbf{A}_{M E M}^{i}, \mathbf{B}^{i}\right)$, we can straightforwardly compute the respective Viterbi-path solving the linear programming step of the FEM-BV-procedure (see Step 2 of the FEM-BV-algorithm description on page 23 of Horenko [15]). Since it is a linear minimization problem 
with convex constraints, it has a unique solution. That is one can uniquely recover the distinct Viterbi path $\Gamma(t)=\left(\gamma_{1}(t), \ldots, \gamma_{k}(t)\right)$ knowing only the full data series $\mathbf{x}_{t}$ and preserving only

$$
N_{\text {param }}=\left(K \star\left(n+M E M \star n^{2}+n \star m\right)+1\right)
$$

parameters while preserving the value of persistency $C$ (here $C=0$ ). Having computed the Viterbi path, one can also compute the distinct values of model errors $\tilde{\epsilon}_{t}^{i}$ as $\tilde{\epsilon}_{t}^{i}=\gamma(i, t) \star\left(\mathbf{x}_{t}-\mu^{i}-\sum_{j=1}^{M E M} \mathbf{A}_{j}^{i} \mathbf{x}_{t-j \tau}-\mathbf{B}^{i} u_{t}\right)$ and test that they are indeed normally distributed prior to application of AIC. The sensitivity experiments we describe (see table 1) effectively bound the problem of over-fitting inherent in analyzing atmospheric observational data.

At one extreme the 60PC experiments with lag 4 days (Expts. $1 \& 5$ ) have multiple tens of thousands of degrees of freedom in the fitting model $(36,121)$ and so are therefore in grave danger of overfitting the reanalysis data with $\mathrm{O}\left(10^{5}\right)$ degrees of freedom. Diagonalizing the matrix A radically reduces the number of degrees of freedom of the fitting model at $M E M=5$ to 720 (Expts. $2 \& 6$ ). While these diagonalised experiments are no longer ill-conditioned, this has come at the cost of ignoring all cross communication between dimensions and thus higher order interactions.

At the other extreme, the 10PC experiments (Expt. 4) have only between $220(M E M=1)$ and $1020(M E M=$ 4) free model parameters and are therefore clearly not over-fitted to the reanalysis data, even when allowing for all cross diagonal terms. The cost in this reduced dimensionality is that retaining so few PCs removes all higher order terms in the reanalysis data which can be critical for particular transient coherent processes (for example blocking) and other less prersistent modes of variability such as the PSA in the SH.

Table 1: Dimension reduction strategies including number of free model parameters calculated using Eqns. $12 \& 14$

\begin{tabular}{|c|c|c|c|c|c|c|}
\hline Expt. & Case & Method & number PCs & Diagonalised & $\mathrm{N}^{o}$ of free parameters & \\
\hline & multi: equivalent barotropic & & & & $M E M=1$ & $M E M=5$ \\
\hline 1) & multi-60 & multi-level PCA & $60 \mathrm{PCs}$ & No & 7321 & 36121 \\
\hline 2) & multi-60-1D & multi-level PCA & $60 \mathrm{PCs}$ & Yes & 240 & 720 \\
\hline 3) & multi-20 & multi-level PCA & $20 \mathrm{PCs}$ & No & 840 & 4040 \\
\hline 4) & multi-10 & multi-level PCA & $10 \mathrm{PCs}$ & No & 220 & 1020 \\
\hline & comb: baroclinic - barotropic & & & & & \\
\hline 5) & comb-60 & independent PCA & (60PCs) 20PCs per level & No & 7321 & 36121 \\
\hline 6) & comb-60-1D & independent PCA & (60PCs) 20PCs per level & Yes & 240 & 720 \\
\hline 7) & comb-21 & independent PCA & (21PCs) 7PCs per level & No & 924 & 4452 \\
\hline
\end{tabular}

\section{Results}

Hoskins and Ambrizzi [19] used the Wentzel-Kramer-Brillouin (WKB) refractive index as a function of the local background state to show that the midlatitude jets act as a waveguide for stationary Rossby waves. Branstator [4] subsequently analyzed monthly mean departures from centered 3-month averages of observational data from the NCEP reanalysis V1, as well as ensemble integrations of atmospheric general circulation models, to show that the Northern Hemisphere $(\mathrm{NH})$ jet stream does in fact act as a waveguide. He pointed out that this waveguide allowed for meridional trapping of zonally elongated disturbances enabling a hemispheric teleconnection pattern to form where distant regions can covary. Importantly, he found that disturbances near the mean jets, and in particular across South Asia, are fundamentally different from those that reside in regions where the meridional gradients are weak. One such region of weaker gradients is the mid-Pacific where the PNA, a pattern with distinct meridional orientation, forms. Due to meridional trapping and zonal elongation, zonally oriented teleconnection patterns within the jets are typically observed to be of smaller scale than the meridionally oriented patterns (PNA \& NAO) consisting of a wavetrain of positive and negative geopotential height anomalies. 
Using FEM-BV-VARX and considering a range of reanalysis products, Risbey et al. [41] showed that the cluster states for the Northern Hemisphere domain (their figure 7) exhibit a midlatitude waveguide, spanning much of the hemisphere. Consistent with the CWP mode identified by Branstator [4], they found a wave 5 pattern in the jetstream waveguide, with a high latitude node over northern Europe. Consistent with the study of Ambrizzi et al. [2], O'Kane et al. [38] found a circumglobal wave 3 (polar)-wave 5 (subtropical) pattern in the $\mathrm{SH}$ jet. However, unlike the $\mathrm{NH}$, they found no evidence of meridionally oriented teleconnections in the major ocean basins, rather a strengthening of the prevailing regional component of the SH equivalent of the CWP, i.e. the PSA in the South Pacific for example.

We present the results in the following logical order and by hemisphere, begining with the NH and considering the equivalent barotropic multi experiments followed by the baroclinic-barotropic comb experiments. Within each of these classes, we first consider the high dimensional (60 PCs) case, then the diagonalised variant followed by each of the non-diagonal reduced PC cases. For non-stationary problems there is no formal criteria to determine where ill-conditioning occurs, instead we make the practical choice to call any patterns that either display no discernable coherent structure, or where the composite structures do not correspond to any plausible observed physical teleconnection pattern, ill-conditioned. We note that composite patterns falling into the aforementioned category only occur at lags $>1$, where many retained dimensions are considered i.e. precisely the cases for which one expects ill-conditioning to occur.

\subsection{Northern Hemisphere}

\subsubsection{Equivalent barotropic}

Using the data reduction strategy outlined in section 2.4, simultaneous multi-level calculation of the leading PCs restricts the dynamics to equivalent barotropic processes.

-multi-60: We first consider the high dimensional experiment where the FEM-BV-VARX is applied to the leading 60 PCs calculated from the multi-level $Z_{g}$ anomalies. In Figure 1, we show annual anomalous $S A T$ and $Z_{g 500 h P a}$ composite cluster patterns. We note that composites for $Z_{g 300 h P a} \& Z_{g 700 h P a}$ are very similar to $Z_{g 500 h P a}$ and will not be shown. For the Bernoulli (lag 0 - optimal) and non-Markovian cases (lag 4), the meridional dipolar structures in the mid-Pacific (PNA) and Atlantic (NAO) are evident. Here the zonal $u$ winds are maximum over South East Asia extending into the western Pacific between $20^{\circ} \mathrm{N}-40^{\circ} \mathrm{N}, 0^{\circ} \mathrm{E}-180^{\circ} \mathrm{E}$ (not shown). The NAO pattern persists and is even stronger at longer lag whereas the PNA pattern degrades somewhat. Anomalous high (low) pressure regions are associated with warm (cold) temperature anomalies at the surface. Despite the likely fact that the non-Markovian case (lag 4 i.e. 5 days memory) would be highly illconditioned the dominant perisitent $\mathrm{NH}$ teleconnections of the PNA and NAO are clearly manifest. The residence times, i.e. time resident in state 1 or 2 , for the seasonal composites (not shown) are highly assymetric in the summer and winter tending to favour one state over the other. This is not the case for the transition seasons, where the residence percentages are relatively evenly split between states.

-multi-60-1D: We next reduce the dimensionality via diagonalization. At lag 0 the annual, and dominant summer and wintertime composite patterns (Figure 2) reveal the zonally oriented hemispheric wave 5-7 pattern. This wavetrain is most prevalent in the North American - Atlantic sector and to a lesser extent in the Pacific. At lag 1 (optimal), the hemispheric wavetrain pattern collapses and is replaced by the meridionally oriented PNA and NAO patterns previously observed at lag 0 for the non-diagonalised case multi-60. For the multi-60-1D case, we expect ill conditioning not to play a role as the dimensionality has been drammatically reduced via diagonalization.

In Figure 3, we show the corresponding annual composite (metastable) states associated with transient baroclinic instability $(\mathrm{PCr})$ and the zonal jet structure $\left(u_{300 \mathrm{hPa}}\right)$. For lag 0 , baroclinic instability is shown to occur in the localised regions of shear between the $Z_{g 300 h P a}$ nodes of the hemispheric wavetrain and zonally oriented in the corresponding regions of large gradients of the $u_{300 h P a}$ winds typically associated with the jet. In contrast, at lag 1 (optimal) baroclinic instability manifests alternating quadrapole structures about the dipolar PNA and NAO patterns largely located where the minimum and maximum $u_{300 \mathrm{~Pa}}$ wind values occur. 
a) z500 (contours) : SAT (shaded) : Northern Hemisphere lag0*
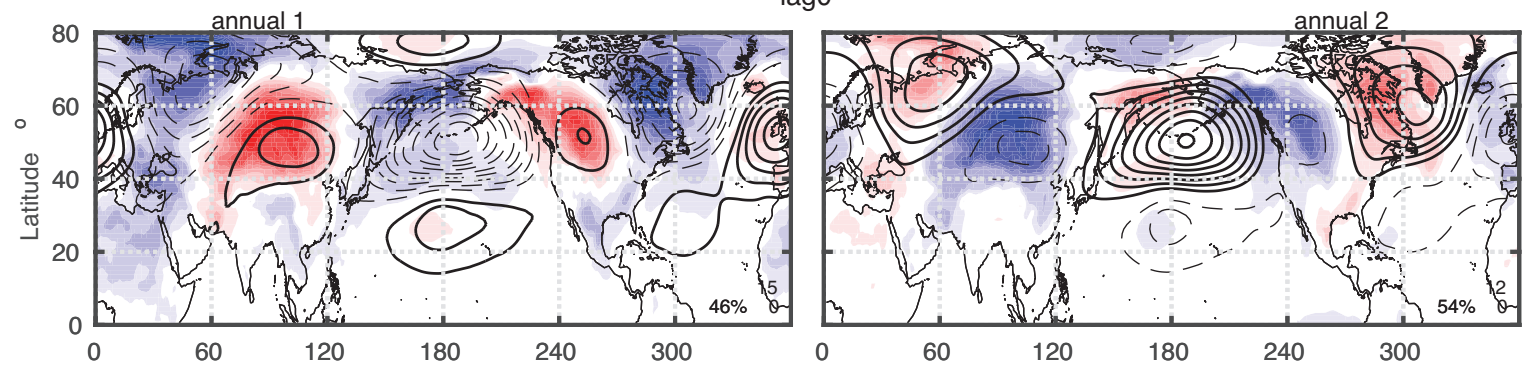

b)

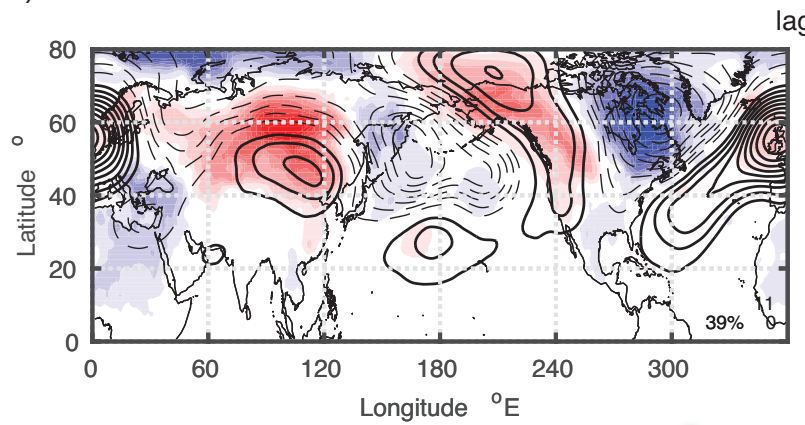

lag4

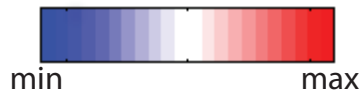

Figure 1: multi-60 : $Z_{g 500 h P a}$ anomaly cluster composite annual states (contours) and $S A T$ (shaded) for a) Bernoulli (lag 0) and b) non-Markovian (lag 4) cases. The fields are partitioned according to the lower colour bar (solid (red)-positive values; dashed (blue)-negative values) and the absolute $\mathrm{max} / \mathrm{min}$ values for the contoured (top value) and shaded (bottom value) fields are shown in the bottom right corner of each of the panels. The percentages indicate time resident in each state. The optimal model according to the Akaike Infromation Criteria is indicated by a star i.e. here the optimal model is for lag0 marked by a star i.e. lag0*.We will follow this convention in all subsequent NH figures.

-multi-20: Reducing the dimensionality further we next consider retaining only the leading 20 PCs (Figure $4 \mathrm{a} \& \mathrm{~b}$ ) however, unlike the diagonalizing approximation, still allowing for communication across dimensions. Annual composites for $Z_{g 300 h P a}$ anomalies and $u_{300 h P a}$ winds show the hemispheric wave train. In contrast to the multi-60-1D case, here we see the Pacific sector wavetrain of similar strength to the North America - Atlantic sector and some indication of the Arctic Oscillation (AO) at high latitudes. The PNA and NAO patterns again emerge at longer lag with lag 2 now optimal.

- multi-10: Retaining only the leading 10 PCs the annual composite states (Figure $4 \mathrm{c} \& \mathrm{~d}$ ) reveal a contraction of the CWP to between $120^{\circ} \mathrm{E}-360^{\circ} \mathrm{E}$. The non-Markovian lag 4 is now optimal with the wavetrain further contracting to being localised over the North American continent. Note that for 10 PCs the results show only wavetrains with no evidence of dipoles, even at long lag. This indicates that in order to capture the persistent, meridionally oriented structures of the PNA and NAO higher order modes modes and memory are required.

In general for the multi-cases, we find that for high dimensionality with communication across all dimensions (multi-60) the NAO and PNA patterns are manifest and the optimal lag is the Bernoulli model (lag 0). As the dimensionality is systematically reduced the CWP at first emerges as a Bernoulli process (multi-60-1D) with the NAO and PNA occurring for the optimal Markov model (lag 1) and non-Markovian models. Further dimension reduction (multi-20) strengthens the CWP as the dominant process at short lag and sees the NAO and PNA occuring where the optimal lag (lag 2) moves to longer timescales. Severe reduction of the dimensionality (multi-10) sees the CWP dominant at all timescales with the optimal lag occurring for the longest 
a) z500 (contours) : SAT (shaded) : Northern Hemisphere

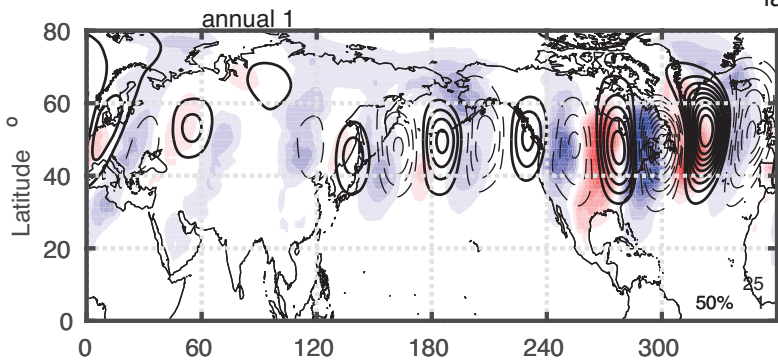

lag0

b)
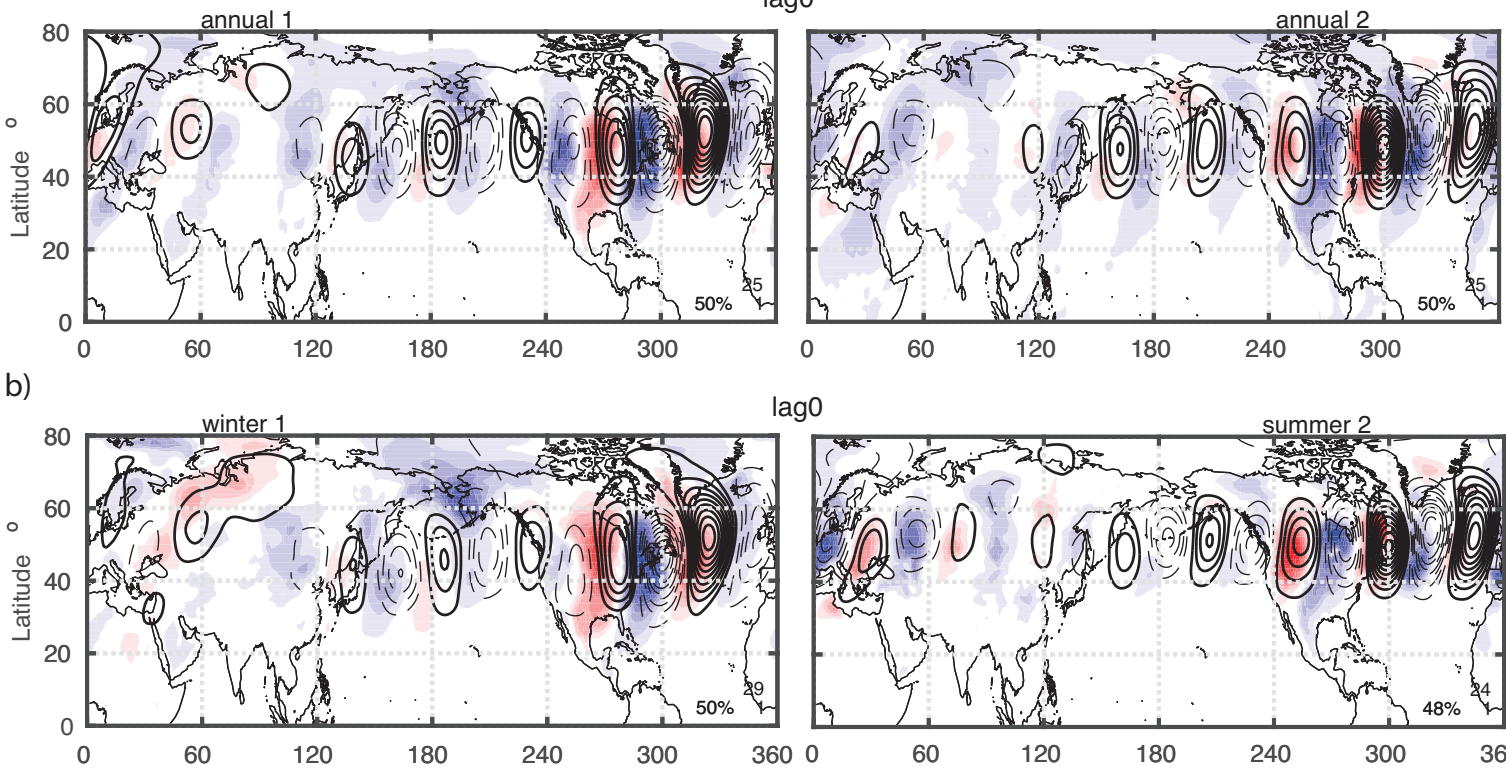

lag0

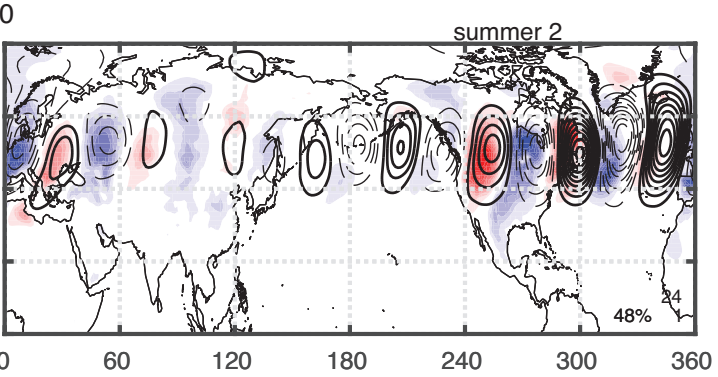

c)

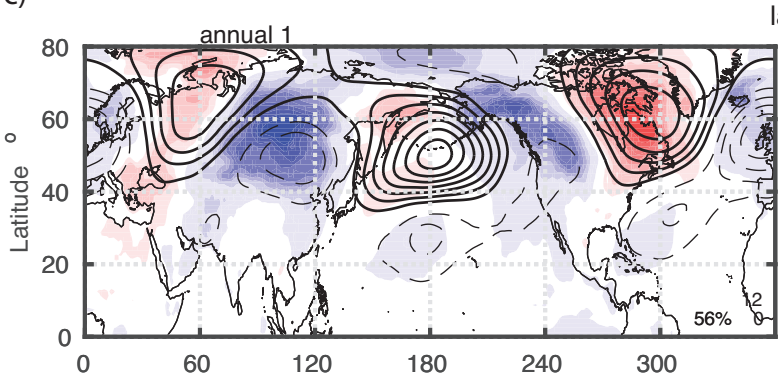

$\operatorname{lag} 1^{*}$

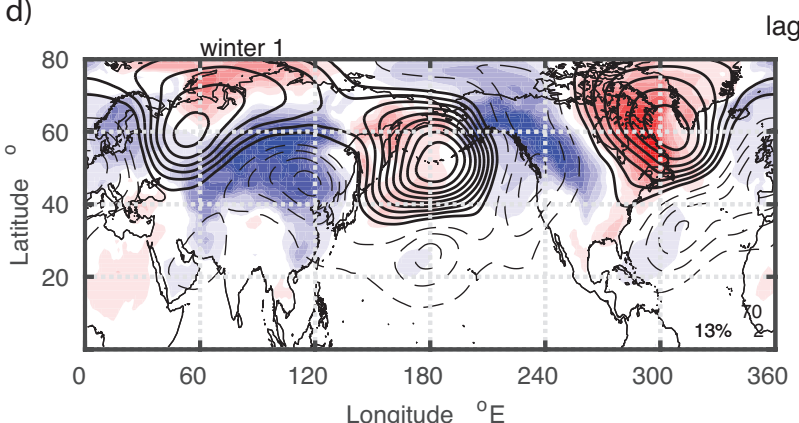

$\operatorname{lag} 1^{*}$

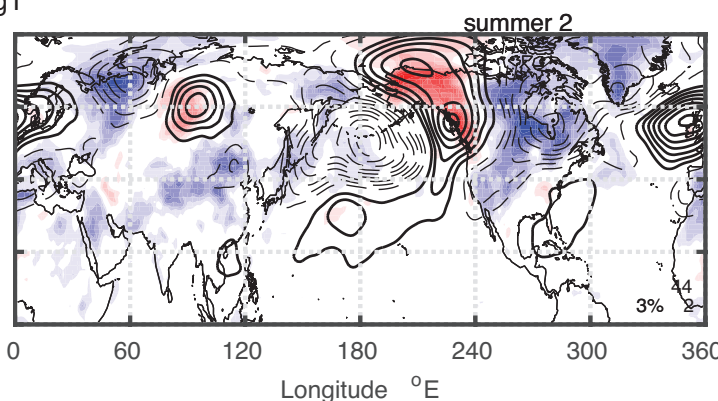

Figure 2: multi-60-1D : Annual and preferred winter and summertime $Z_{g 500 h P a}$ anomaly cluster composite states (contours) and $S A T$ anomalies (shaded) for lags 0 and 1.

memory model (non-Markovian) considered (lag 4). Generally the PNA and NAO are high dimensional persistent teleconnections whereas the CWP is the low dimensional more transient mode.

\subsubsection{Baroclinic - barotropic}

Combining the leading 20PCs calculated independently for each level, we allow for baroclinic as well as barotropic processes. 
a) u300 (contours) : PCr anomaly (shaded) : Northern Hemisphere
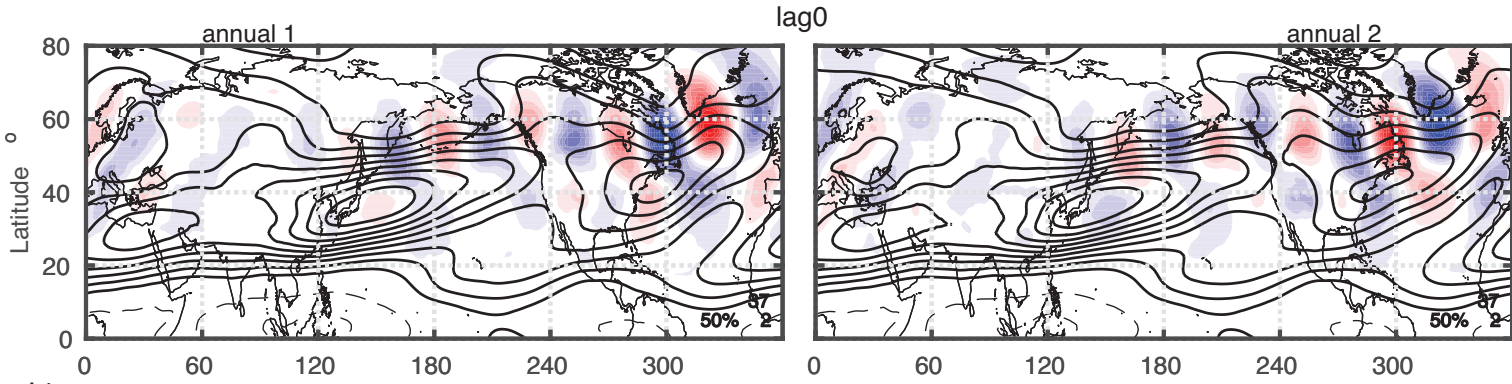

$$
\text { b) }
$$

$\operatorname{lag} 1^{*}$
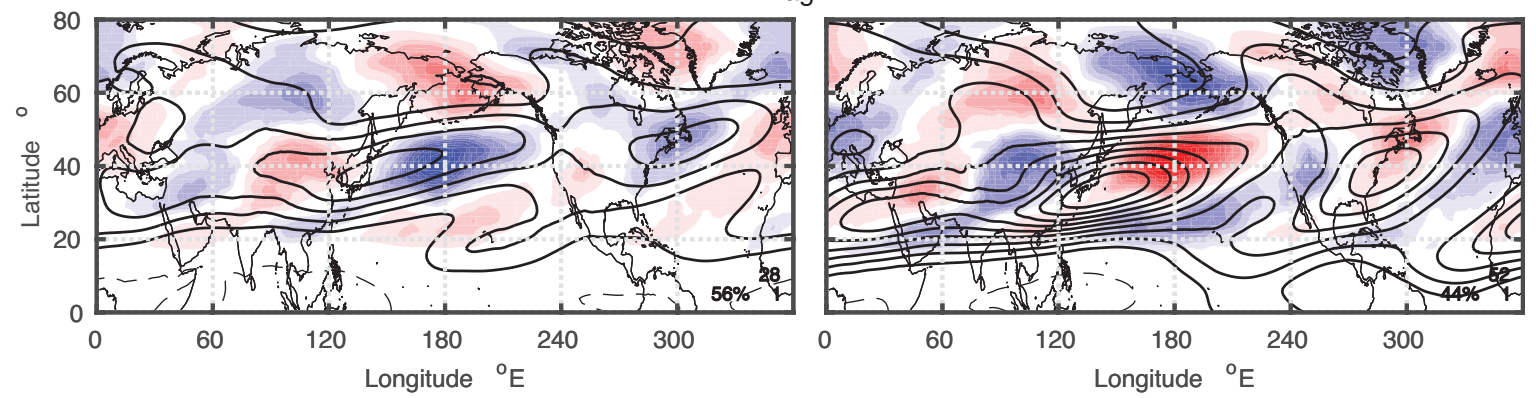

Figure 3: multi-60-1D : Annual $P C r$ (shaded) anomaly cluster composite states and mean $u_{300 h P a}$ winds (contour) for lags 0 and 1.

- comb-60: Here, and at all lags, we observe the PNA and NAO (annual) patterns (figure 5). This is much like the patterns observed in the multi-60 case, but more clearly resolved. As with the earlier multi-60 case, there is a large assymetry in residence time, i.e. a tendency to lock into one phase, during winter and particulalrly summer but less so during the transition seasons. Particularly large cold/warm SAT anomalies occur over the North American continent at all lags for states 1 and 2 - here associated with large persistent negative and positive height anomalies.

-comb-60-1D: Diagonalizing the independent PCs reveals aspects of the CWP - in particular weak wavetrains over Eurasia and the central Pacific Ocean at lag 0 (optimal) and a much more intense wavetrain over North America - North Atlantic. As the lag increases, the Pacific basin wavetrain strengthens until it becomes dominant at lag 4 (Figure 6). Here, we show only the annual composites as the seasonal patterns are very similar to those for the multi-60-1D lag0 case. In contrast to the multi60-1D cases where wavetrains are only observed for the Bernoulli models, for comb-60-1D we can infer that even the more persistent baroclinicbarotropic structures that form on the nodes of the NH CWP wavetrain, for example blocking structures, do not require cross communication of the leading modes as long as baroclinic processes relevant to onset and decay of blocks are present.

- comb-21: Reducing the number of PCs to consider only the leading seven PCs at each level (Figure 7) reveals zonally oriented dipolar structures in the Pacific (associated with blocking in the Gulf of Alaska) and over the North East of the USA and into the Atlantic. These dipolar structures are much larger than the associated regional CWP nodes and, although zonally oriented, are in regions normally associated with the meridional PNA and NAO patterns. Here, we show only the annual states for the optimal lag 2 as the other lags have essentially the same features also present in all seasons. The large zonally oriented dipolar structures present over the North Atlantic and Pacific are absent in any of the multi-calculations which display either the CWP or meridionally oriented dipoles in these sectors. As the multi- experiments are by construction equivalent barotropic, one might argue that the zonal dipolar structures evident here arise due to baroclinicity in these sectors indicating more equally important contributions from barotropic and baroclinic instability to the formation and persistence of the particular PNA and NAO coherent structures. 
z500 (contours) : u300 (shaded) : Northern Hemisphere
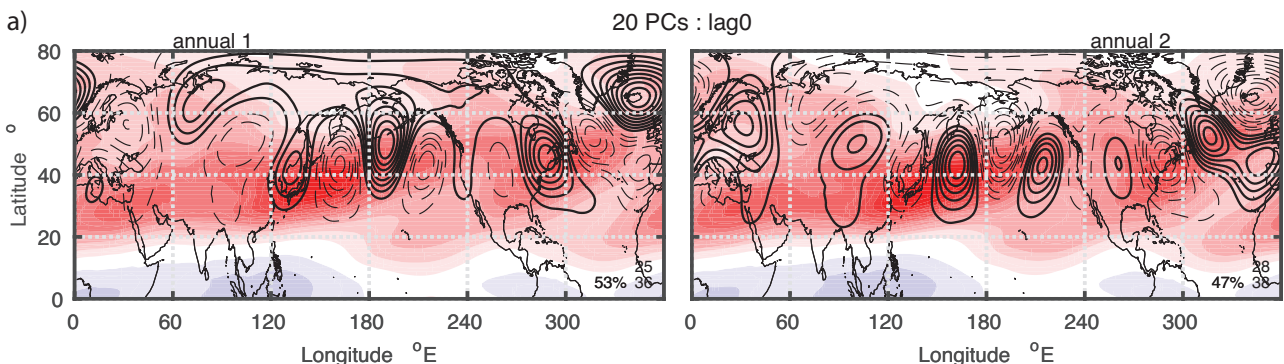

z500 (contours) : SAT (shaded) : Northern Hemisphere

b)

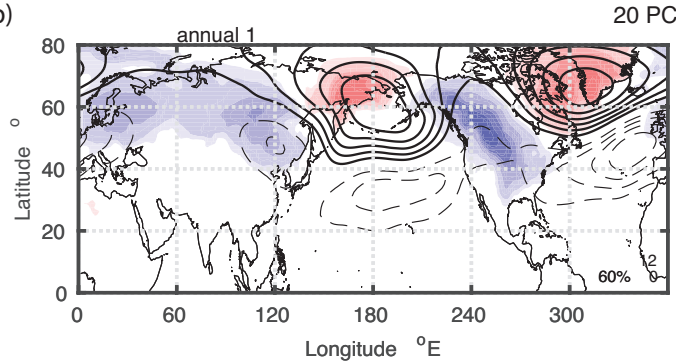

20 PCs : lag2

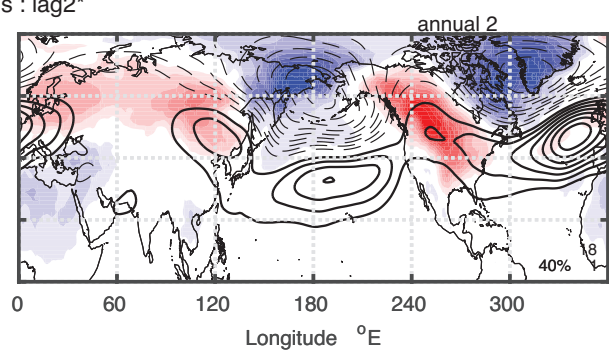

z500 (contours) : u300 (shaded) : Northern Hemisphere

c)
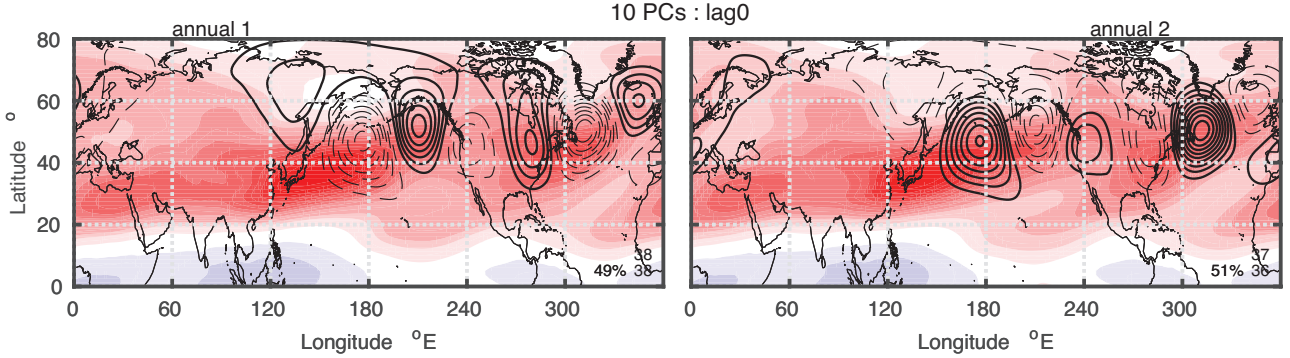

z500 (contours) : SAT (shaded) : Northern Hemisphere

d)
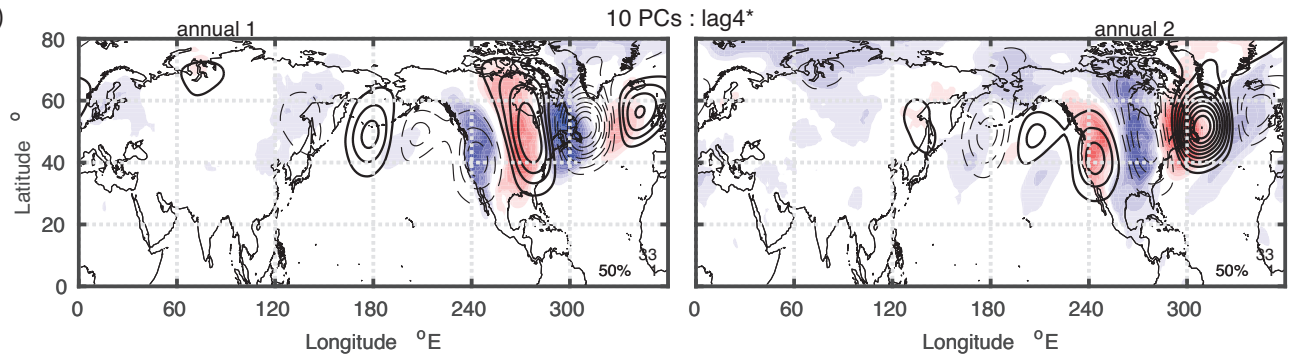

Figure 4: a) \& c) annual $u 300 h P a$ winds (shaded) and $Z_{g 500 h P a}$ anomaly (contours), b) \& d) $S A T$ anomaly cluster composite states (shaded) and $Z_{g 500 h P a}$ anomaly (contours) at 0 and the optimal lag for multi-20 (a) \& b)) and multi-10 (c) \& d)) cases respectively.

\subsection{Southern Hemisphere}

\subsubsection{Equivalent barotropic}

O'Kane et al. [38] used FEM-BV-VARX to examine the dynamical drivers of systematic changes in persistent quasi-stationary states (regimes) of the Southern Hemisphere (SH) troposphere at 500hPa. They found composite states for the hemisphere corresponding to a circumglobal wave 3 (polar)-wave 5 (subtropical) pattern, 

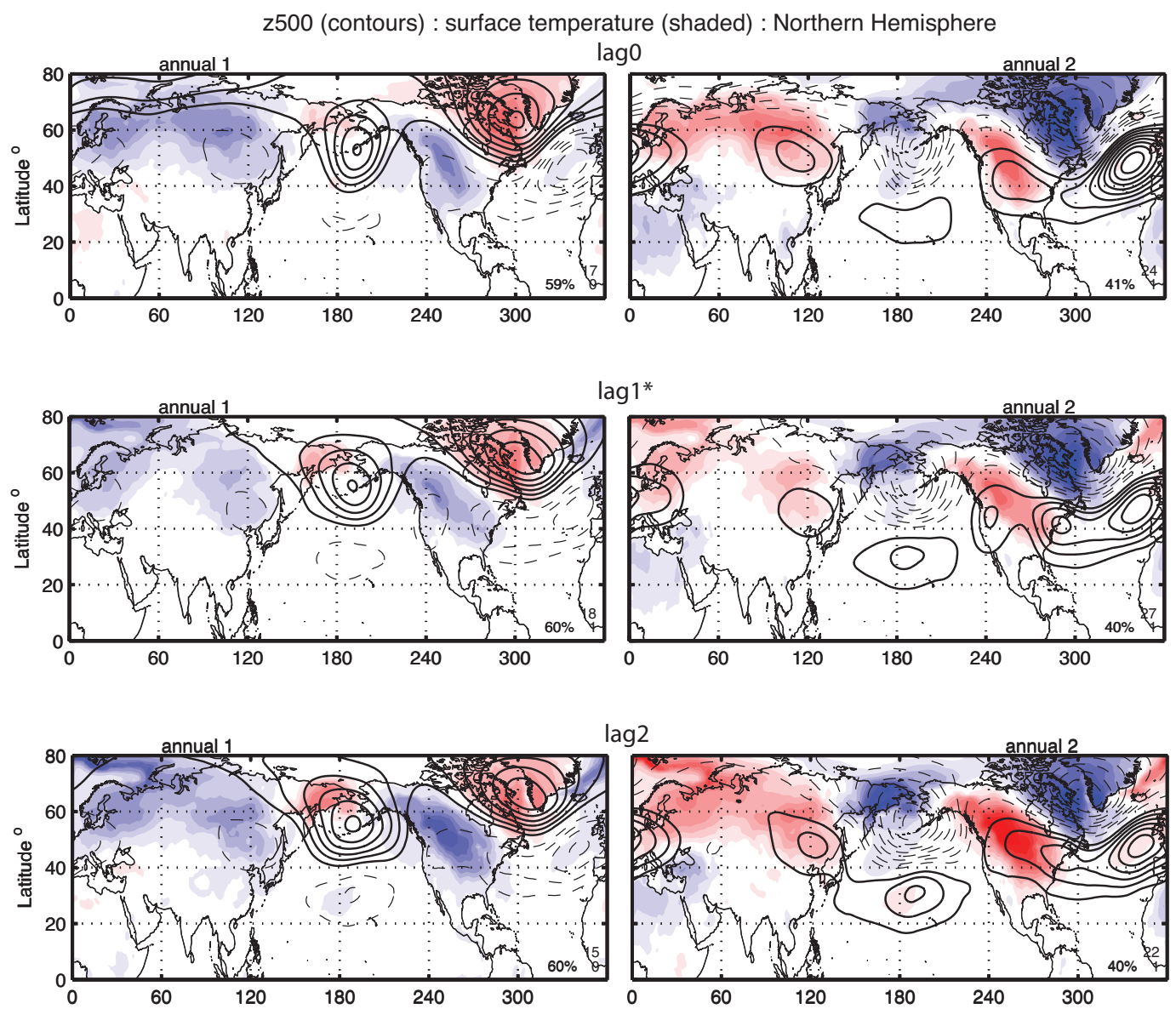

lag2
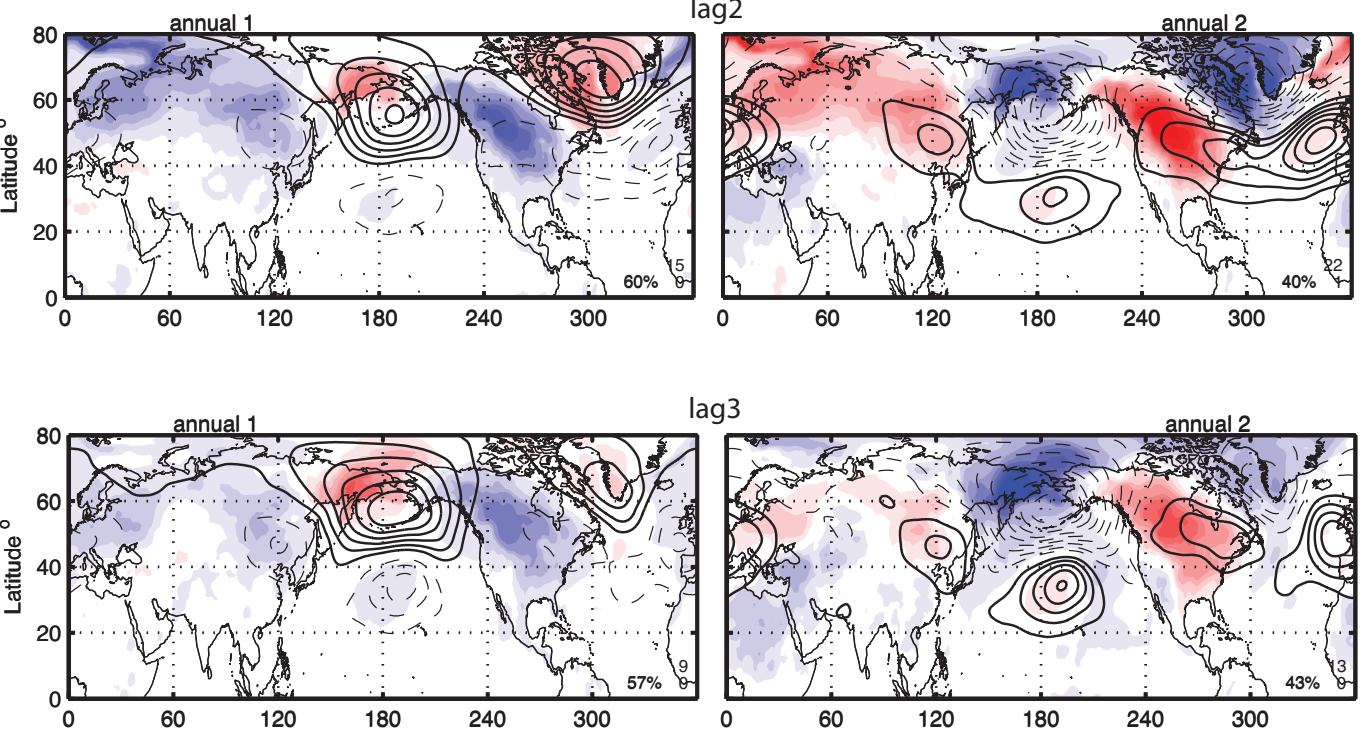

lag3
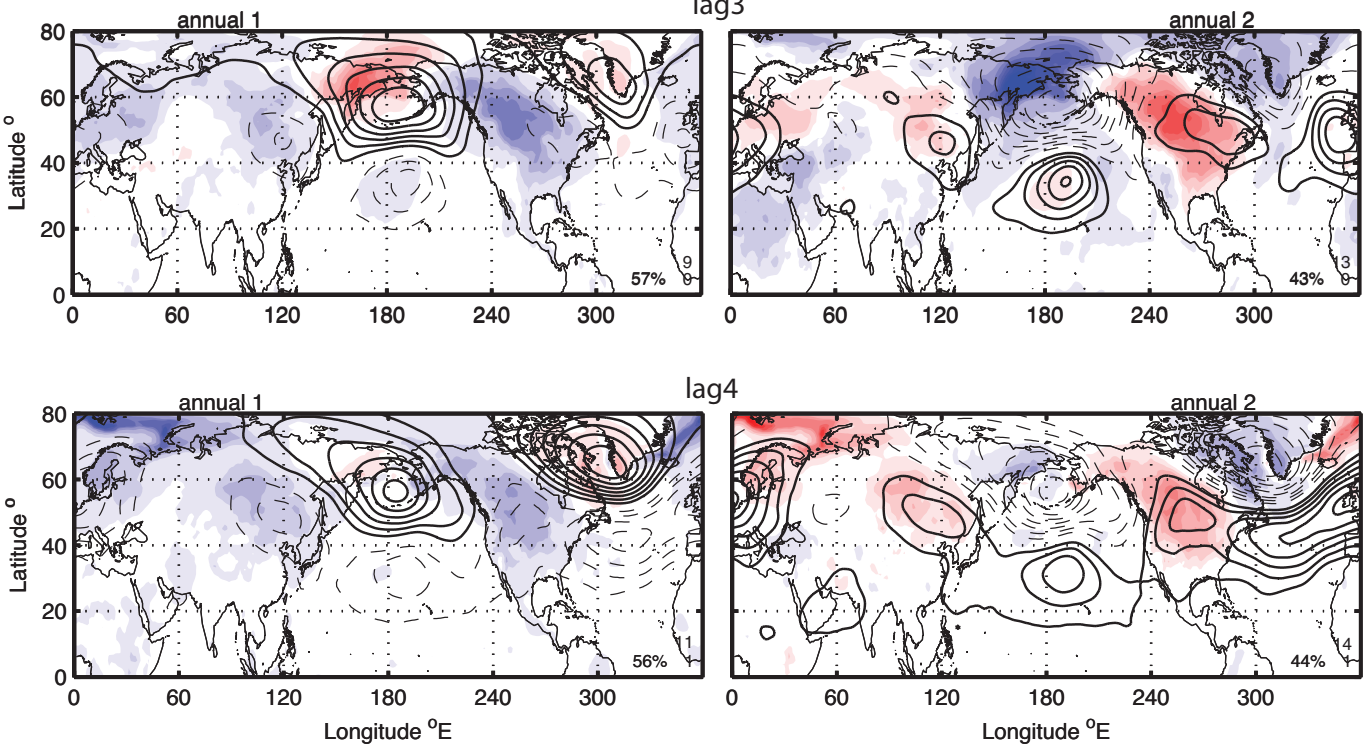

lag4

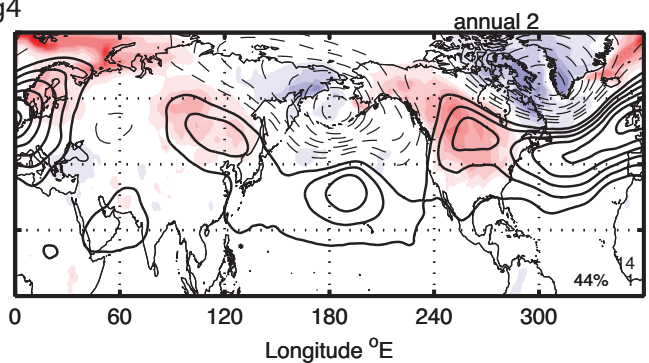

Figure 5: comb-60: Annual $Z_{g 500 h P a}$ anomaly cluster composite states (contours) and $S A T$ anomalies (shaded) for lags 0-4.

while regional composites revealed the PSA pattern and blocking modes. They further found that regional blocking and teleconnection patterns (PSA) largely correspond to amplification of sections of the zonal circumglobal SH wavetrain with little evidence of meridional orientation. Here, we extend that study O'Kane 

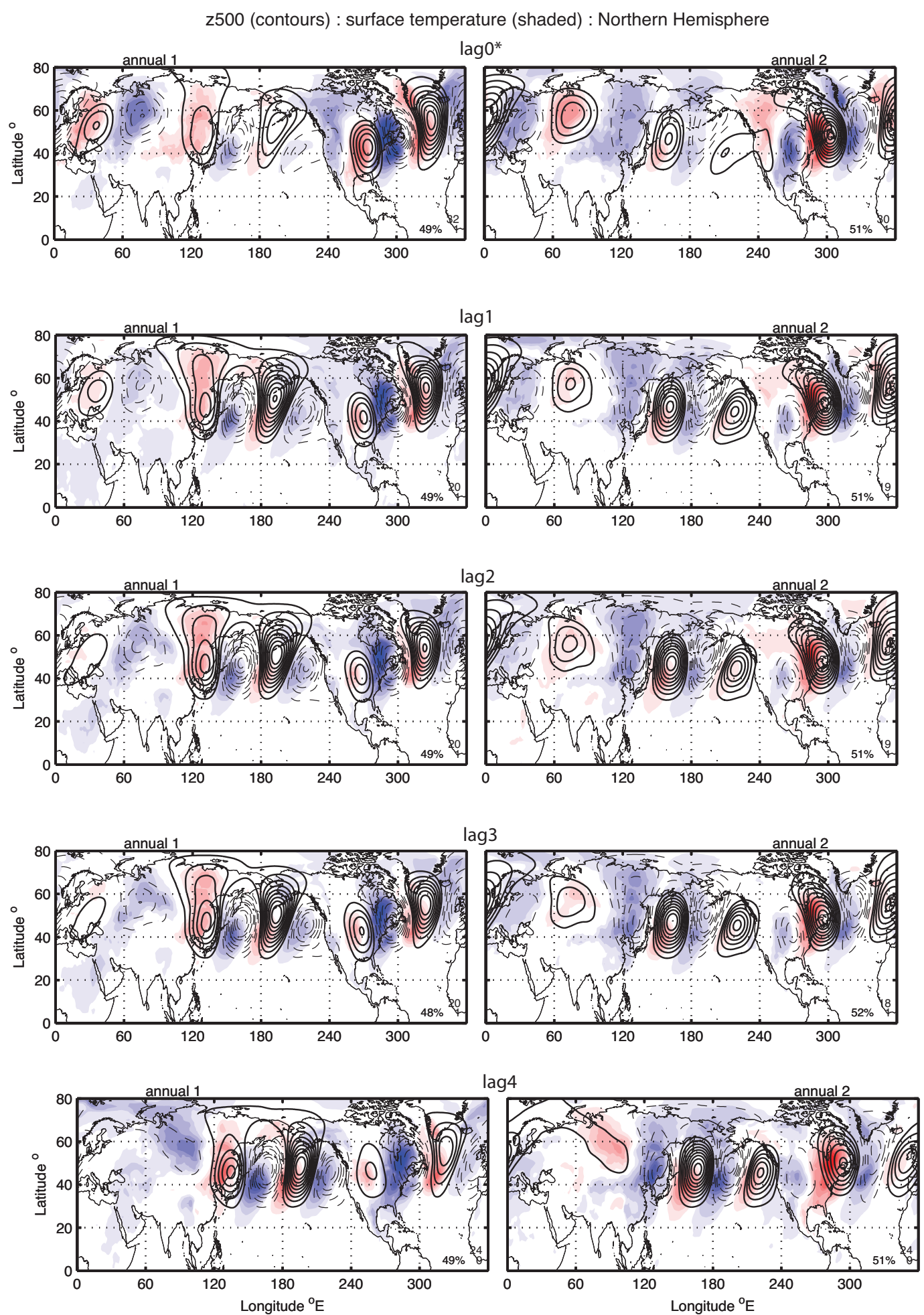

Figure 6: comb-60-1D : Annual $Z_{g 500 h P a}$ anomaly cluster composite states (contours) and $S A T$ anomalies (shaded) for lags 0-4.

et al. [38] to consider the 3D structure of the $\mathrm{SH}$ troposphere and to explore the conditionality of the flow regimes. We first examine composite patterns derived from applying FEM-BV-VARX to the PCs describing SH multi-level equivalent barotropic processes. 


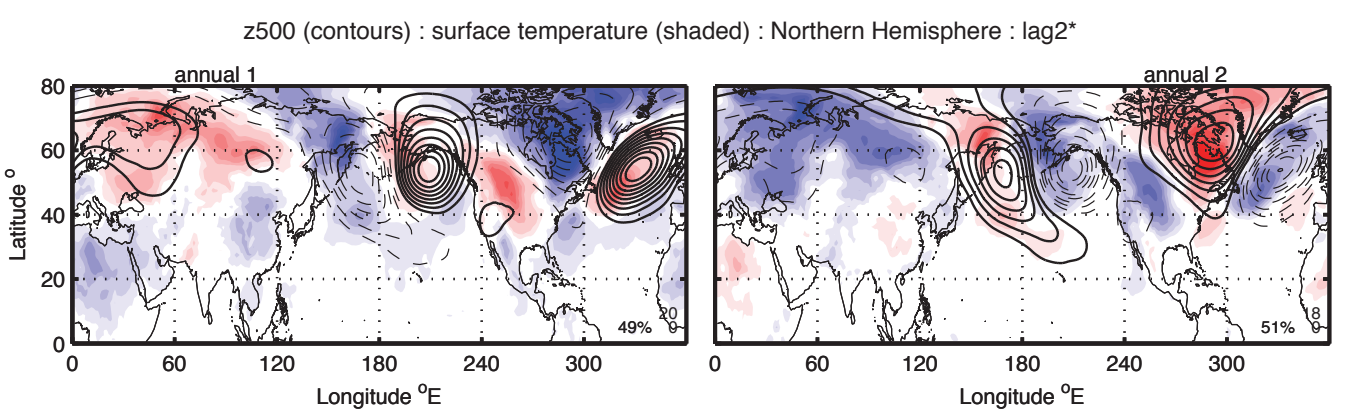

Figure 7: comb-21: Annual $Z_{g 500 h P a}$ anomaly cluster composite states (contours) and $S A T$ anomalies (shaded) for optimal lag 2.

- multi-60: In Figure 8, we show $Z_{g 500 h P a}$ composite states obtained from the multi-60 experiment where the FEM-BV-VARX is applied to the leading $60 \mathrm{PCs}$ calculated from the multi-level $Z_{g}$ anomalies over the SH. For the annual patterns at lag 0 (optimal), the midlatitude wave 3 pattern and high latitude zonal structure typical of the SAM are present. As the memory retained, i.e. lag, is increased there is evidence of a degree of disruption to the SAM annual patterns indicating some degree of ill-conditioning.

\section{z500 (contours) : SAT (shaded) : Southern Hemisphere}
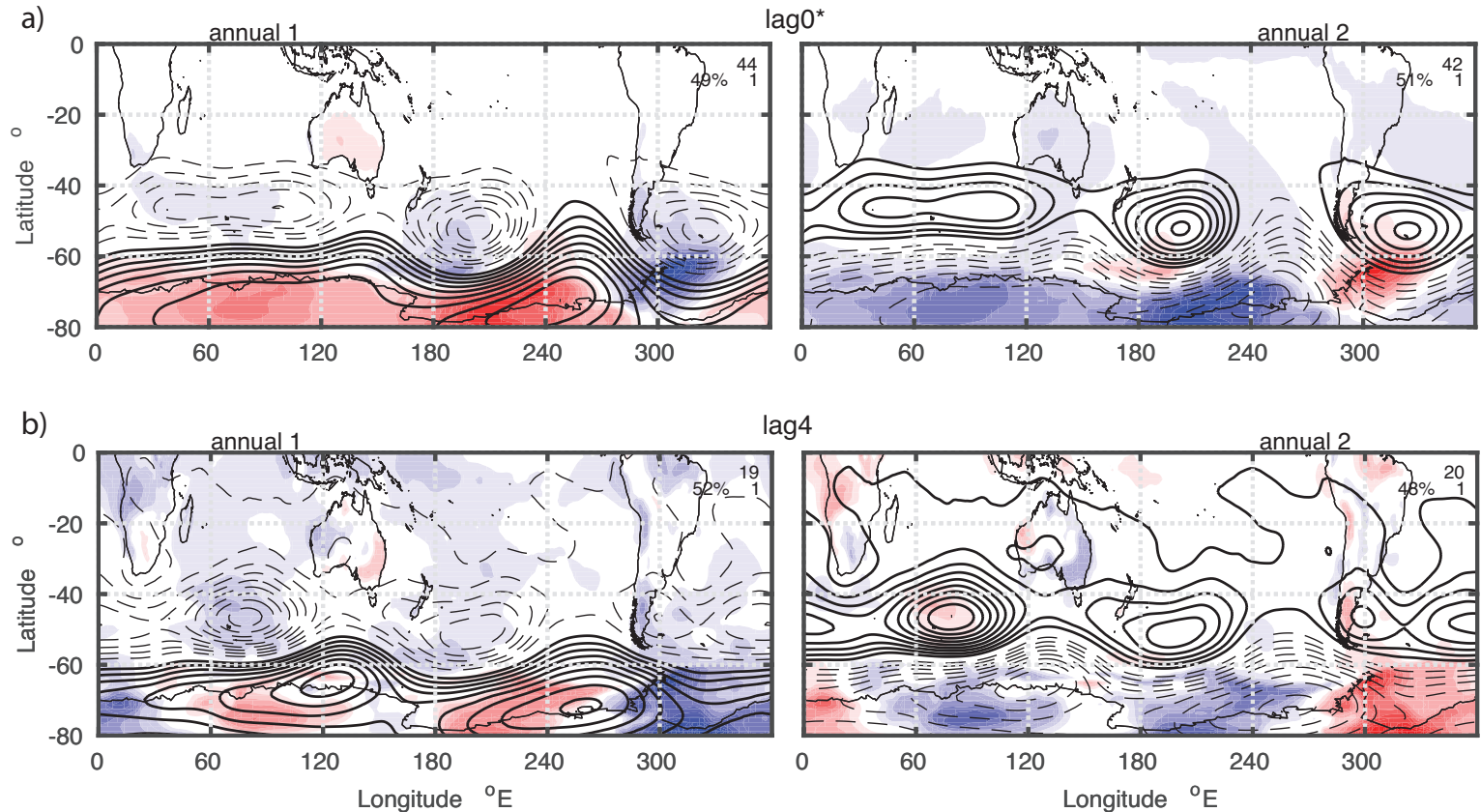

Figure 8: multi-60: $Z_{g 500 h P a}$ anomaly (contours) and $S A T$ anomalies (shaded) cluster composite annual states for a) Bernoulli (lag 0) and b) non-Markovian (lag 4) cases. The fields are partitioned according to the colour bar in figure 1: (solid (red)-positive values; dashed (blue)-negative values) and the absolute max/min values for the contoured (top value) and shaded (bottom value) fields are shown in the top right corner of each of the panels. The percentages indicate time resident in each state. The optimal model according to the Akaike Infromation Criteria is indicated by a star i.e. here the optimal model is for lag0 i.e. lag0*.We will follow this convention in all subsequent SH figures.

-multi-60-1D: Applying the diagonalizing approximation (Figure 9) reveals the SH CWP evident at lag 0 (optimal) and contracting to the Indian Ocean sector at longer lags as seen in the $Z_{g 500 h P a}$ annual composite states (note that $Z_{g 300 h P a}$ and $Z_{g 700 h P a}$ composite states are qualitatively the same). Here, we show only 
the annual patterns in $Z_{g 500 h P a}$. However, we note that the wintertime subtropical jet influences the CWP strongly at lag 0 and lag 1, largely manifesting as elongated and amplified nodes of the CWP in the Tasman Sea and South Pacific Ocean sectors $\left(120^{\circ} \mathrm{E}-300^{\circ} \mathrm{E}\right)$. In contrast, the summertime circulation is more uniformly zonal. As memory effects (lag) are incorporated, the Indian Ocean sector becomes increasingly dominant in all seasons. This accords with the fact that the meridional gradients of the jet waveguide are most pronounced in the Indian Ocean sector.

z500 (contours) : SAT (shaded) : Southern Hemisphere

a)

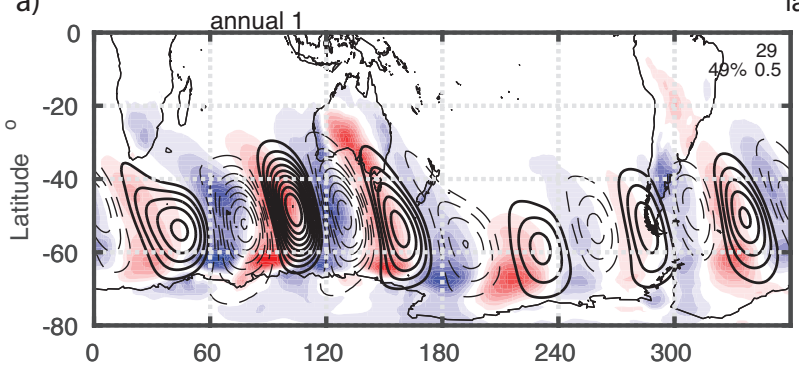

$\operatorname{lag} 0^{*}$

b)

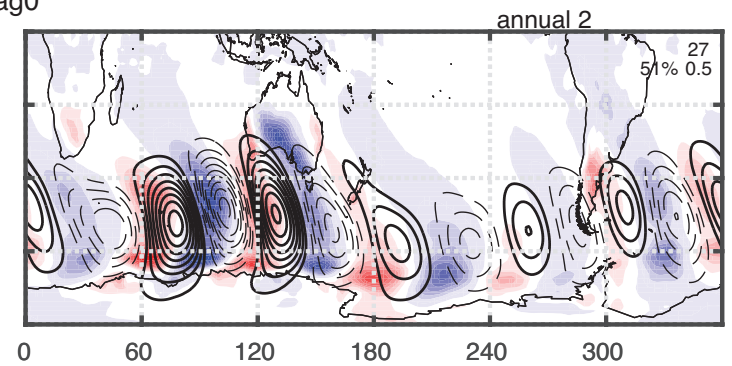

$\operatorname{lag} 4$
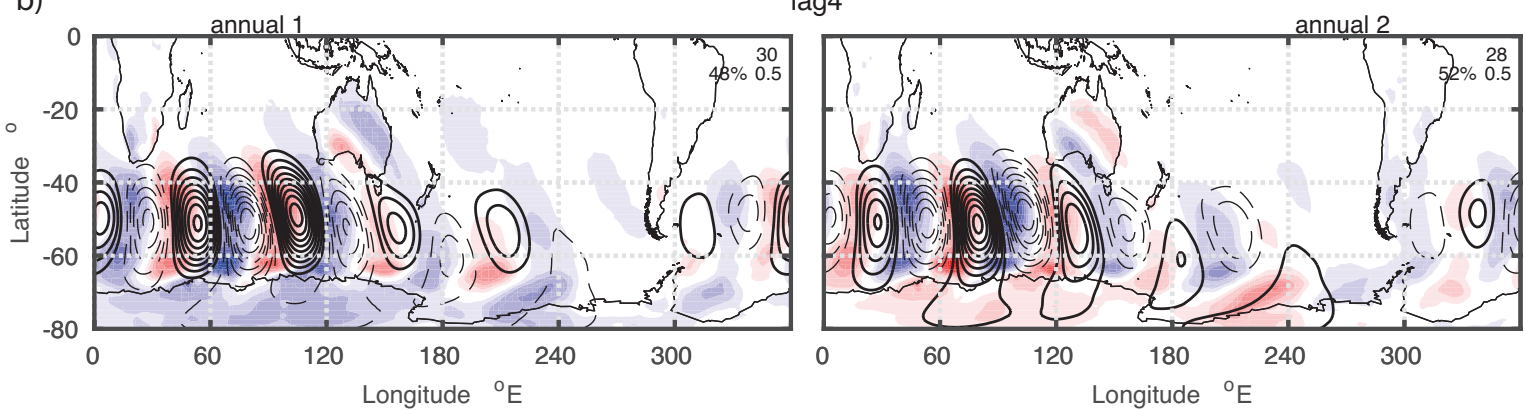

Figure 9: multi-60-1D : Annual $Z_{g 500 \mathrm{PPa}}$ anomaly cluster composite states (contours) and $S A T$ anomalies (shaded) for lags 0 and 4.

Annual composite patterns of baroclinic instability (Figure 10), with the mean $u_{300 h P a}$ winds superimposed for lag 0 (optimal) and lag 4 consistently reveal regions of large gradients in $u_{300 h P a}$ in the Indian Ocean sector as the primary source of coherent baroclinic instability, typically associated with the formation of coherent synoptic disturbances. Wintertime patterns (not shown) reveal a clear splitting of the subtropical and polar jets, regions of increased equatorward instability due to the presence of the subtropical jet, and a PSA $2[33,36]$ type pattern in $\mathrm{PCr}$ in the South East Pacific. At longer lags, baroclinic instability is most evident in the Indian Ocean, indicating that the polar jet in the Pacific does not play a significant role on the dynamics of memory dependent persistent states. For the summertime (not shown), the circulation is largely zonal, where the significant meridional gradients of the poleward side of the polar jet are the primary source of instability. Although weak in comparison to the wintertime, the CWP is most evident only at lag 0-1, manifesting only in the Indian Ocean sector at longer lags.

- multi-20: Reducing the dimensionality to the leading $20 \mathrm{PCs}$, composite preferred winter and summertime patterns of $Z_{5500 \mathrm{hPa}}$ anomaly and $u_{300 \mathrm{hPa}}$ wind composite states at lag 0 (Figure $11 \mathrm{a} \& \mathrm{~b}$ ) clearly show the SH CWP and the influence of the wintertime subtropical $\left(20^{\circ} \mathrm{S}-40^{\circ} \mathrm{S}\right)$ and summertime polar $\left(40^{\circ} \mathrm{S}-60^{\circ} \mathrm{S}\right)$ jets. At the optimal lag 2 (figure $11 \mathrm{c} \& \mathrm{~d}$ ), we observe that the respective nodes of the height anomalies are now resolved within their respective seasonal jets.

-multi-10: Further reducing the dimensionality to only the leading $10 \mathrm{PCs}$, the winter and summertime $Z_{g 500 h P a}$ and $S A T$ composites (Figure 12) at lag 0 reveal structures consistent with the multi-2O and regional blocking patterns coincident with the nodes of the SH CWP and with some larger scale structure in the South 
a)

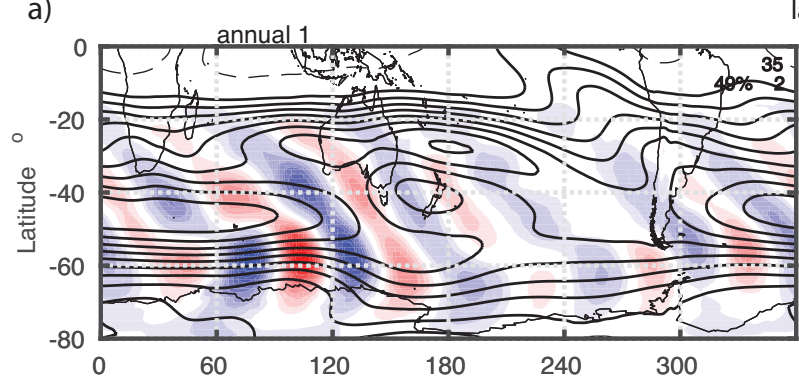

$\operatorname{lag} 0^{*}$ b)

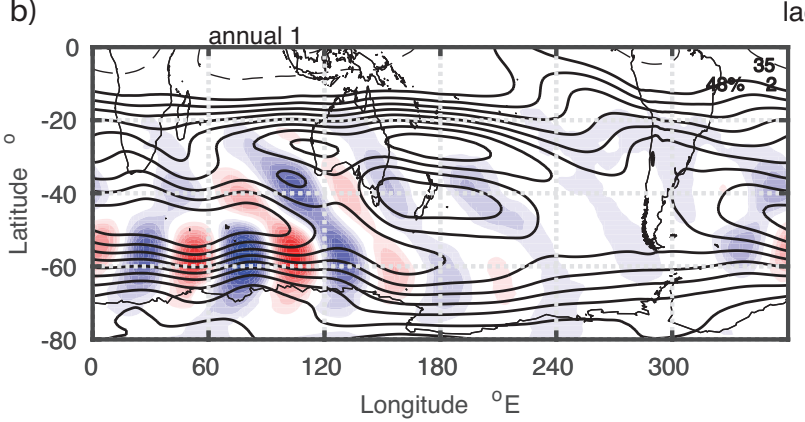

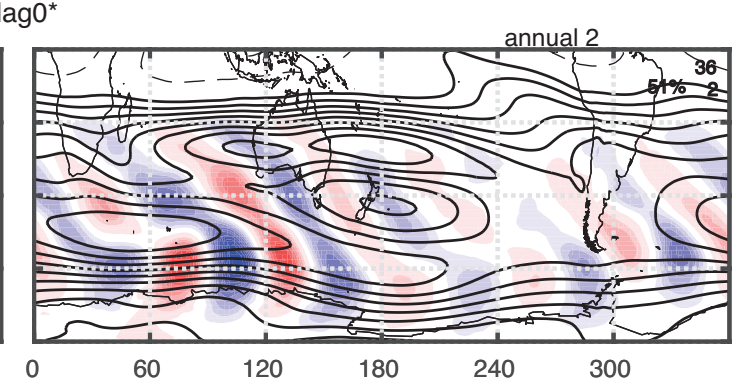

$\operatorname{lag} 4$

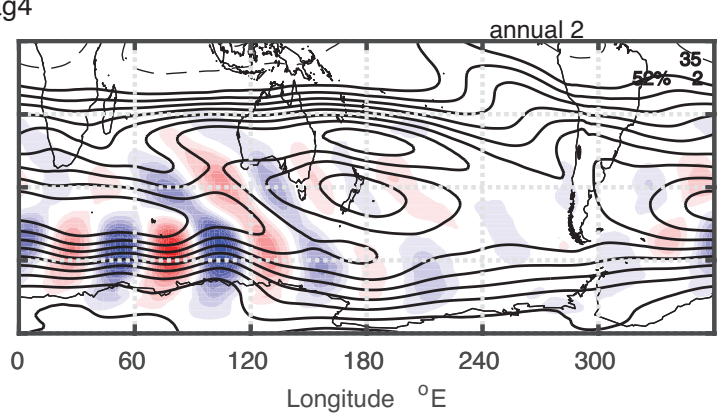

Figure 10: multi-60-1D : Annual $P C r$ anomaly (shaded) cluster composite states and $u_{300 h P a}$ (contours) for lags 0 and 4 .

East Pacific where the PSA pattern occurs. At lag 3 (optimal), the CWP pattern starts to break down. As the dimensionality is low, we do not consider ill-conditioning to play a role here. Rather, it is more likely that the transient nature of the formation and persistence of coherent patterns in the $\mathrm{SH}$ (relative to the $\mathrm{NH}$ ) requires many more modes to be retained to account for a significant amount of the variance. This interpretation would be consistent with the recent study of the PSA, the most persistent SH teleconnection pattern, by O'Kane et al. [36], showing that the variance of SH coherent patterns is widely distributed across multiple scales with little evidence of scale separation required to indicate the existence of a slow manifold.

\subsubsection{Baroclinic - barotropic}

We next examine composite patterns derived from applying FEM-BV-VARX to the PCs describing SH baroclinic - barotropic processes.

- comb-60: Considering the leading 20PCs calculated independently for each level, the CWP evident at lag 0 appears in both the winter and summer (Figure 13), with a clear PSA1 pattern in the South East Pacific. This is in contrast to the NH, where we have only the meridionally oriented patterns (PNA \& NAO) and no CWP (see figure 5). The wintertime PSA pattern, evident at lag 0 , weakens and contracts poleward at lag 1 . The summertime CWP extends uniformly within the polar jet, but significantly weakens at lag 1 , remaining strong only in the Indian Ocean sector, whereas the wintertime CWP has significant extension into the subtropical jet over the Australian mainland. The composite structures at lags $>1$ are highly ill-condtioned (not shown), i.e. as the lag is increased more and more correlations across time are included and the dimensionality increases accordingly. While the CWP - including a strong PSA - constitutes the annual pattern at lag 0 (not shown), only the Indian ocean sector is clearly resolved at lag 1 . Note that the $\mathrm{NH}$ comb-60 case does not appear to be similarly ill-conditioned at longer lag, reflecting the more persistent metastable stuctures of the NH, i.e. PNA and NAO, relative to the more transient SH quasi-stationary states (blocking and the PSA). 
z500 (contours) : u300 (shaded) : Southern Hemisphere

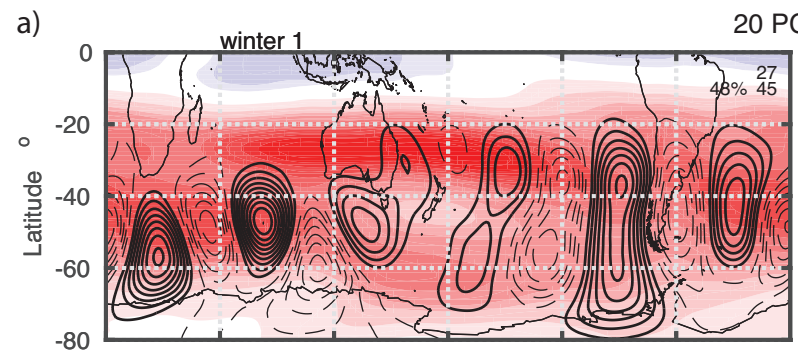

20 PCs : lag0

b)
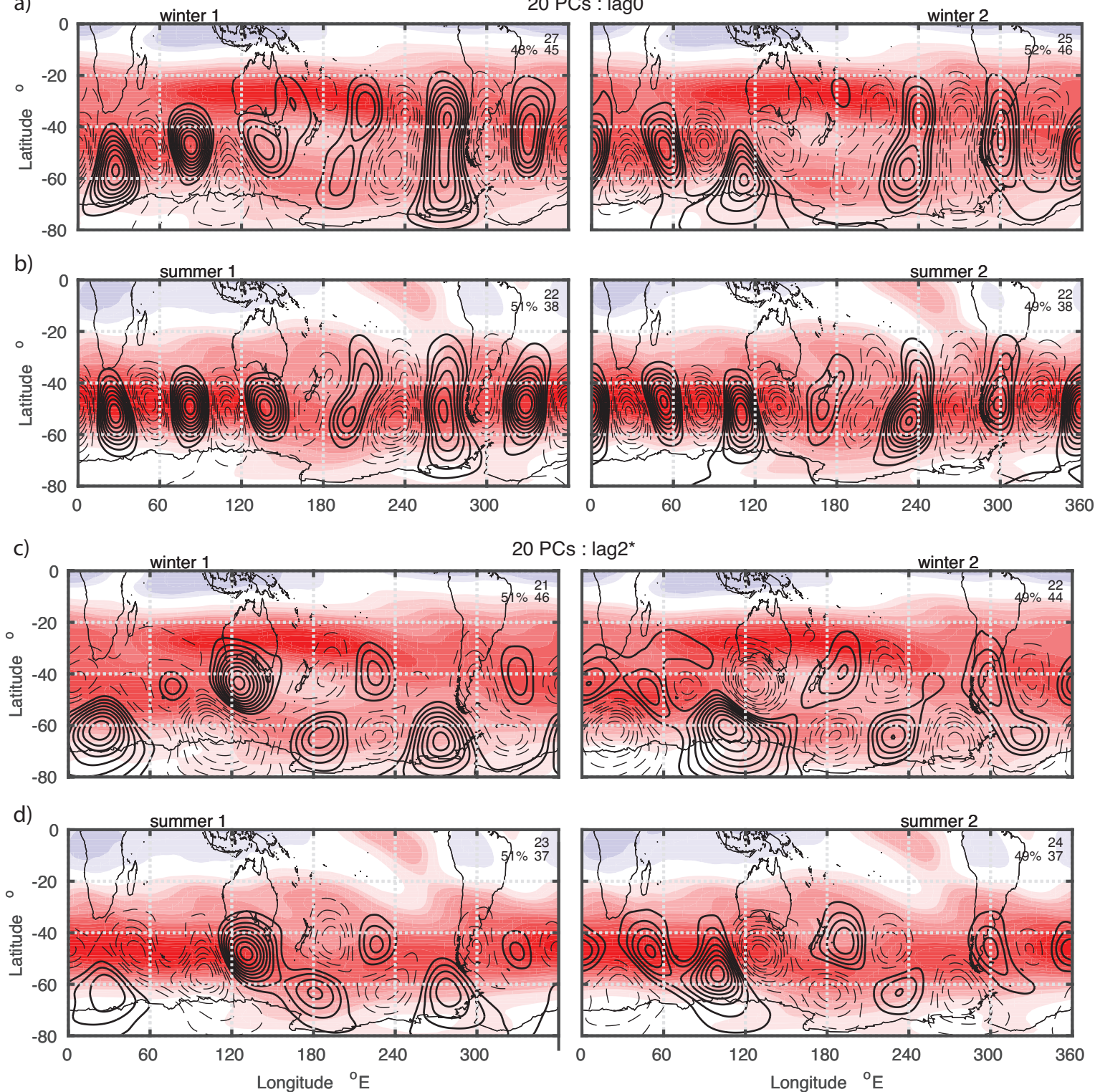

Figure 11: multi-20 : Winter and summer $Z_{g 500 h P a}$ anomaly (contour cluster composite states and $u_{300 h P a}$ (shaded) for lags 0 and 2.

-comb-60-1D: Upon diagonalizing, the CWP, here shown for winter and summer for the optimal lag 0 and at lag 4, manifests at all lags and all seasons (Figure 14). Here we have also superimposed the mean u300 winds indicating the wintertime subtropical jet and summertime polar jet structures.

- comb-21: Reduction to the leading seven PCs at each level (Figure 15), we show only the lag 0 and lag 2 (optimal) winter and summer composite states. We note that beyond lag 2 the persistent patterns do not change significantly. At lag 0 , we see the Indian Ocean dominating both seasons but in particular in the wintertime. In the summer, a weak wavetrain in the Pacific appears due to the strengthened polar jet. For the optimal lag 2, a clear PSA2 pattern is present in the Pacific in both seasons and a clear CWP in the summer. These patterns are somewhat similar to those for comb-60 at lags 0 and 1, although the PSA patterns are in quadrature, i.e. comb-60 resembles PSA1, whereas comb-21 is more closely matched to PSA2. 

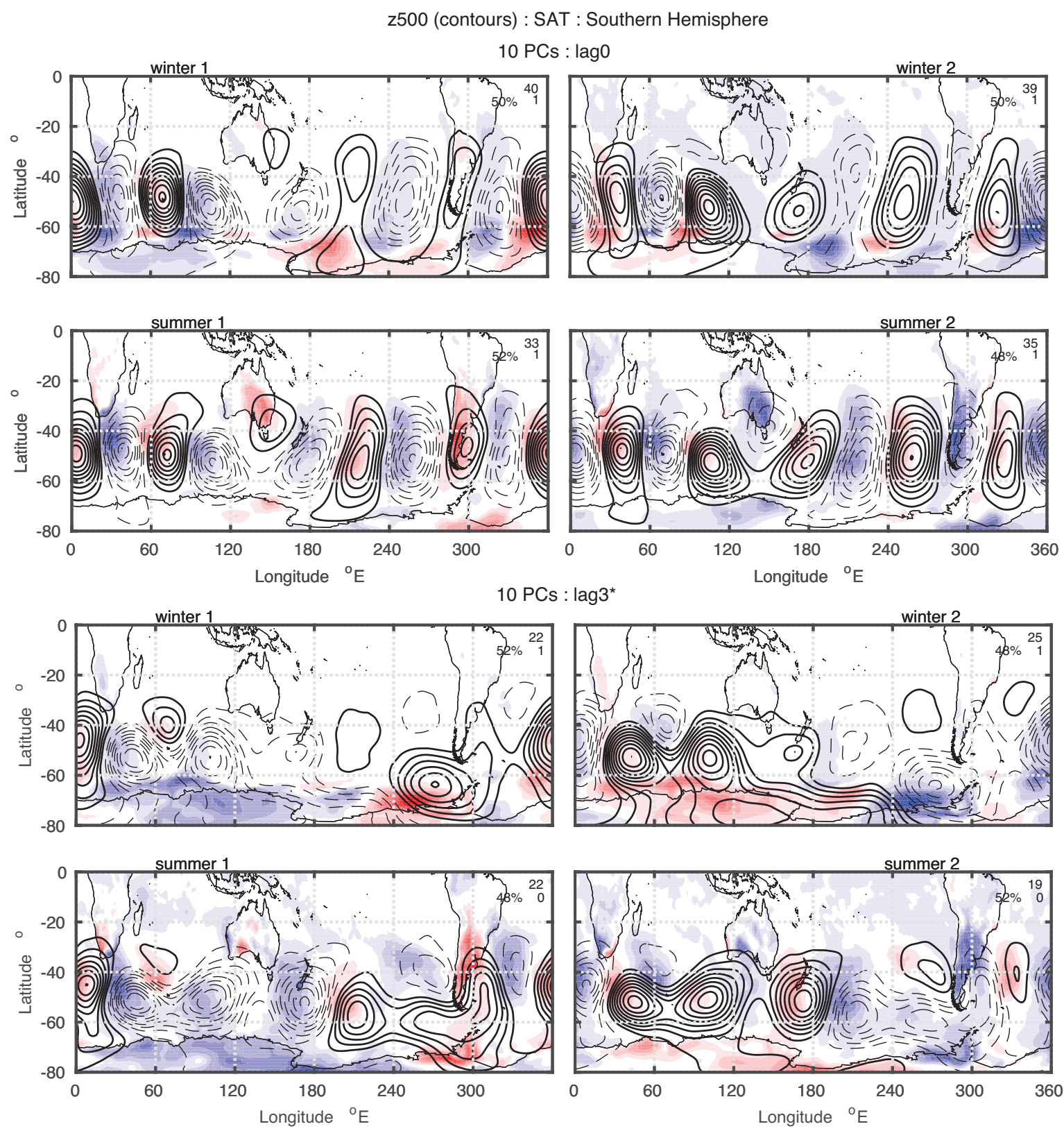

Figure 12: multi-10 :Winter and summer $Z_{g 500 h P a}$ anomaly cluster composite states (contours) and $S A T$ anomalies (shaded) for lags 0 and 3.

\section{Summary}

We summarise each experiment noting only those results that are robust to conditioning and are interpretable in terms of the well known atmospheric teleconnections. We compare and contrast the equivalent barotropic (multi) and baroclinic - barotropic (comb) experiments and the specific high dimensional, diagonalised and reduced dimension cases. 
z500 (contours) : surface temperature (shaded) : Southern Hemisphere

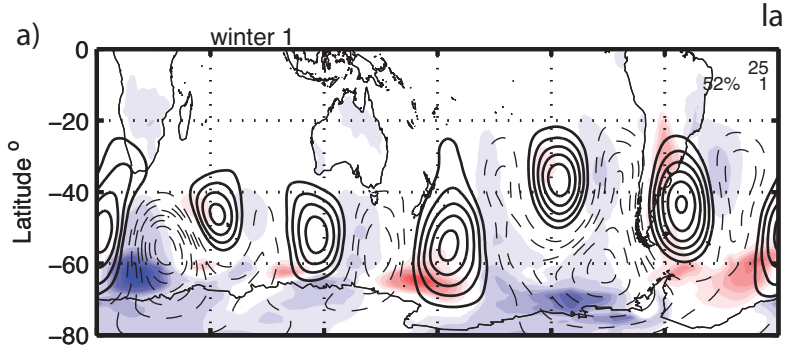

$\operatorname{lag} 0$
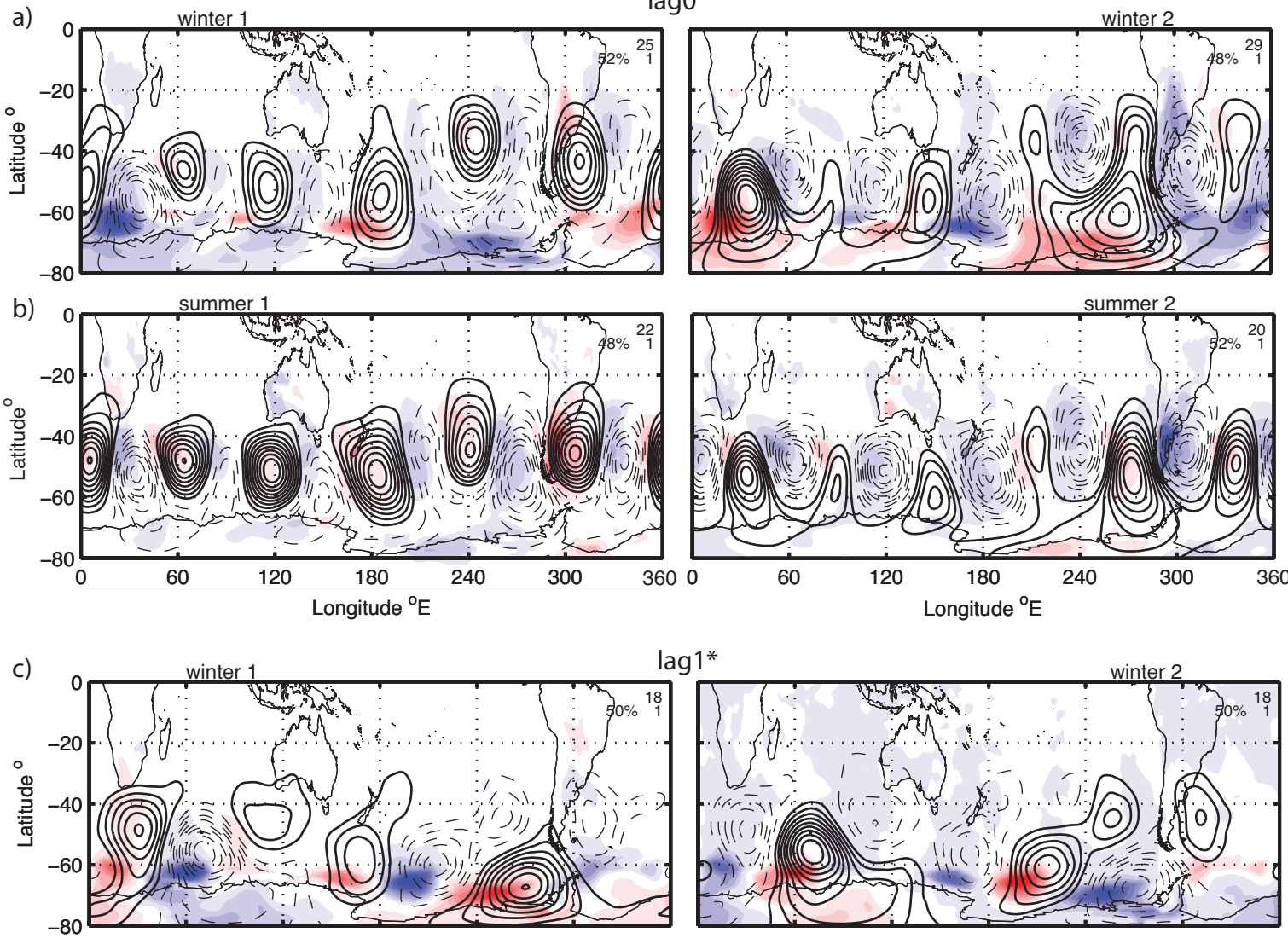

$\operatorname{lag} 1^{*}$
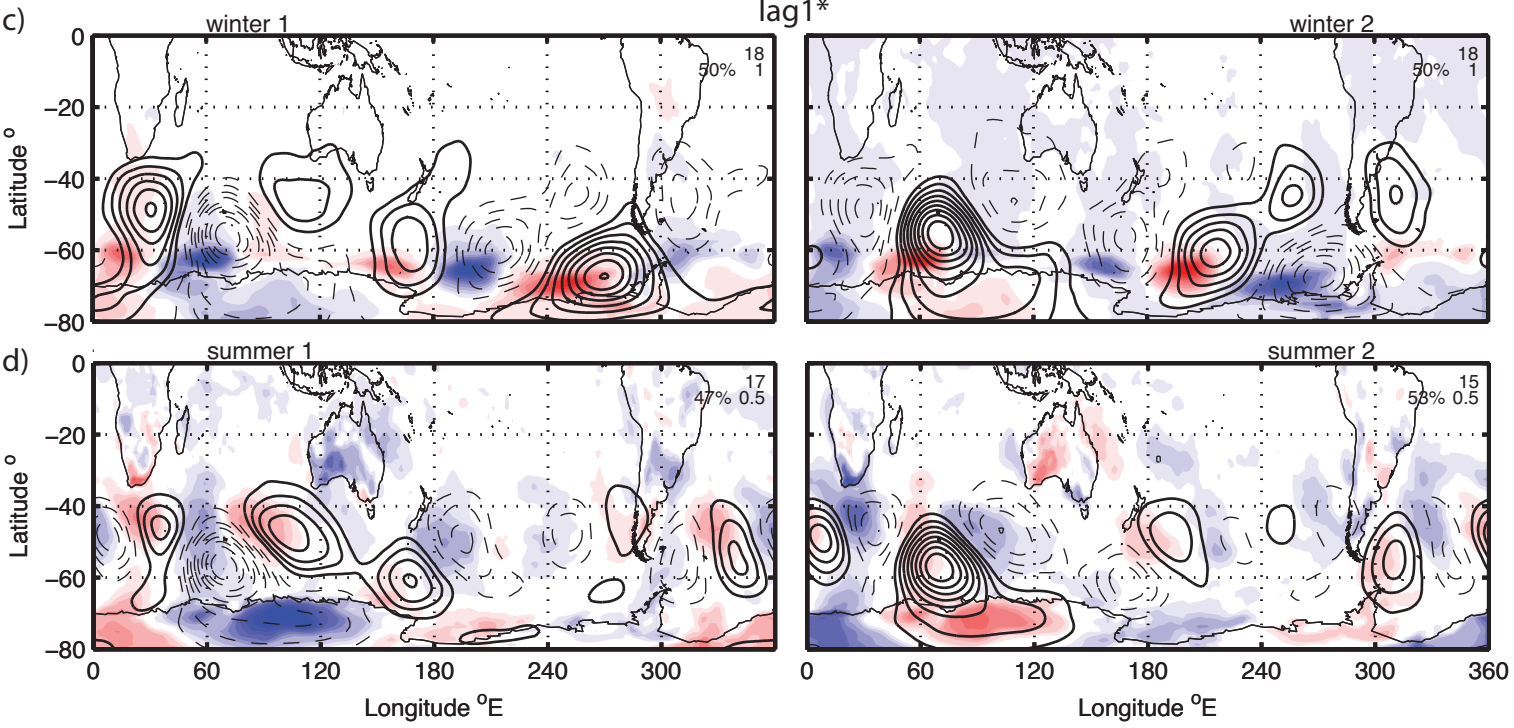

Figure 13: comb-60 : Winter (a \& c) and summer (b \& d) $Z_{g 500 h P a}$ anomaly cluster composite states (contours) and $S A T$ anomalies (shaded) for lags $0-1$.

\subsection{Summary of Northern Hemisphere teleconnections:}

- PNA-NAO meridional teleconnections are present in both the multi-60 and for comb-60 cases and are indicative of the importance of the higher order PCs (dimensions) and their impact on the leading modes of variability.

- Blocking modes appear where the dimensionality is reduced to the leading PCs with coupling across dimensions still allowed or by diagonalization in combination with limited memory, i.e. inclusion of the higher order dimensions supress the zonally oriented wavetrain associated with synoptic activity, while enhancing the large meridionally oriented dipolar modes of the NAO and PNA.

- The CWP is dominant in the Bernoulli model where the diagonalizing approximation is applied, i.e. where there is no communication across the dimensions (multi-60-1D \& comb-60-1D), or where the number of PCs has been substantially reduced and regardless of the initial approach to constructing the covariances 

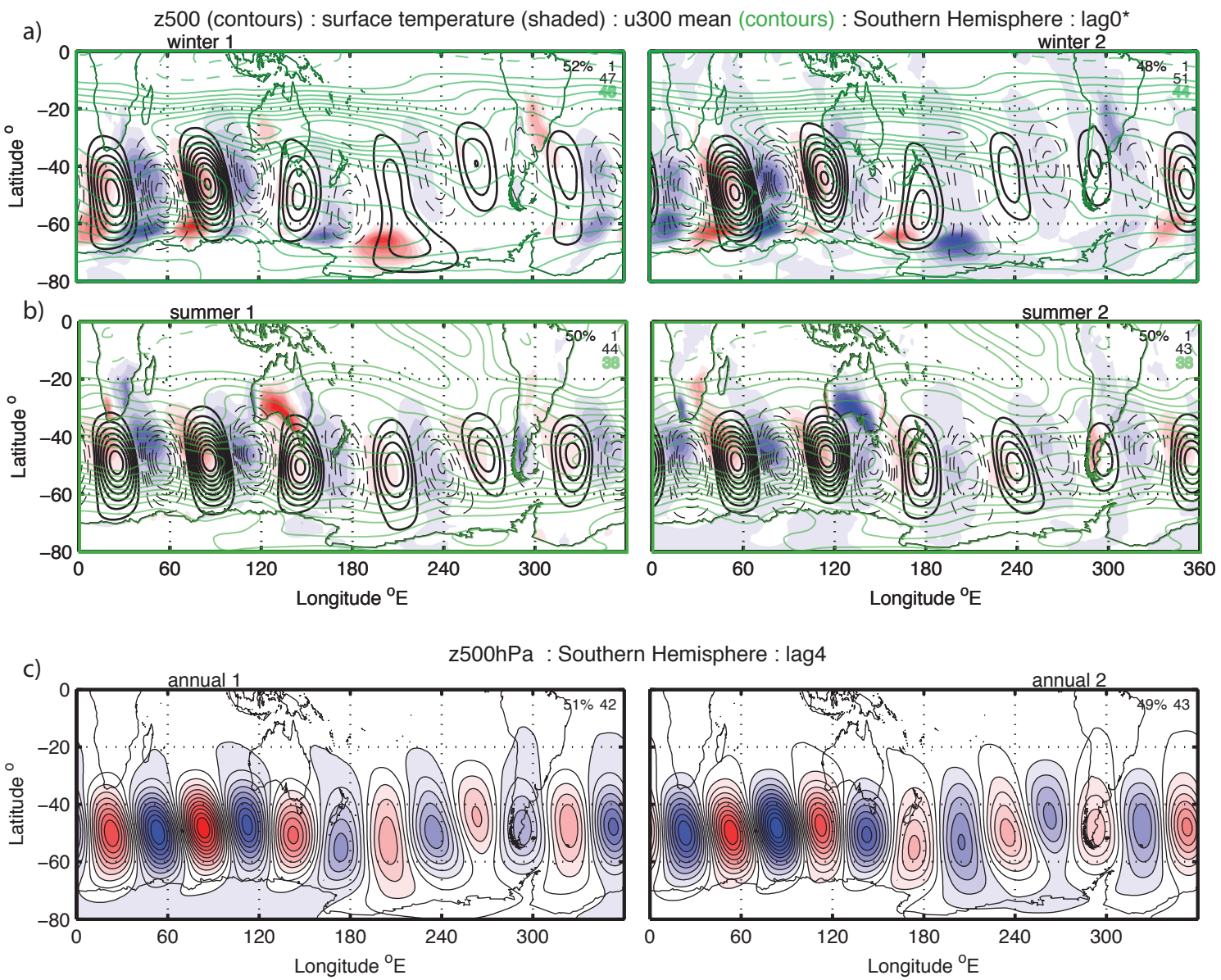

Figure 14: comb-60-1D : Winter (a) and summer (b) $Z_{g 500 \mathrm{hPa}}$ anomaly cluster composite states (black contours), mean $u_{300 h P a}$ winds (green contours) and $S A T$ anomalies (shaded) for optimal lag 0. (c) Annual $Z_{\text {g500hPa }}$ anomaly cluster composite states for lag 4.

be it multi- or comb-. The North American - North Atlantic sector of the CWP dominates, particularly where the number of dimensions has been severely truncated. In general, the PNA and NAO are dominant where long memory is allowed and or a large number of dimensions retained.

- AO emerges strongly where coupling is allowed, i.e. multi-10, multi-20, comb-60 and comb-21.

- Reduction to the leading few (in this case seven) PCs at each independent level (comb-21) represents a severe dimension truncation while still allowing for baroclinic processes. This is in contrast to the equivalent barotropic multi-20 and multi-10 cases. With comb-21 we found large scale zonally oriented dipolar structures in regions normally associated with the meridional PNA and NAO patterns. In comparison to the equivalent barotropic case, we see zonal dipolar structures in the North Atlantic arising due to the inclusion of baroclinicity in that sector whereas the CWP, including blocking over Eurasia, and the PNA and NAO were found in the equivalent barotropic cases. The PNA appears to arise due to the combined effects of barotropic and baroclinic processes.

For the NH equivalent barotropic cases we found that only for the Bernoulli model with high dimensionilty, communication across all dimensions and with memory (multi-60) did the NAO and PNA patterns manifesting at all lags. As the dimensionality is progressively reduced, the CWP emerges first in the Bernoulli model (multi-60-1D), then increasingly at longer lag as the dimensionality is systematically reduced. For severe dimension truncation (multi-10) only the CWP is observed. In general for high dimensional models, as non- 

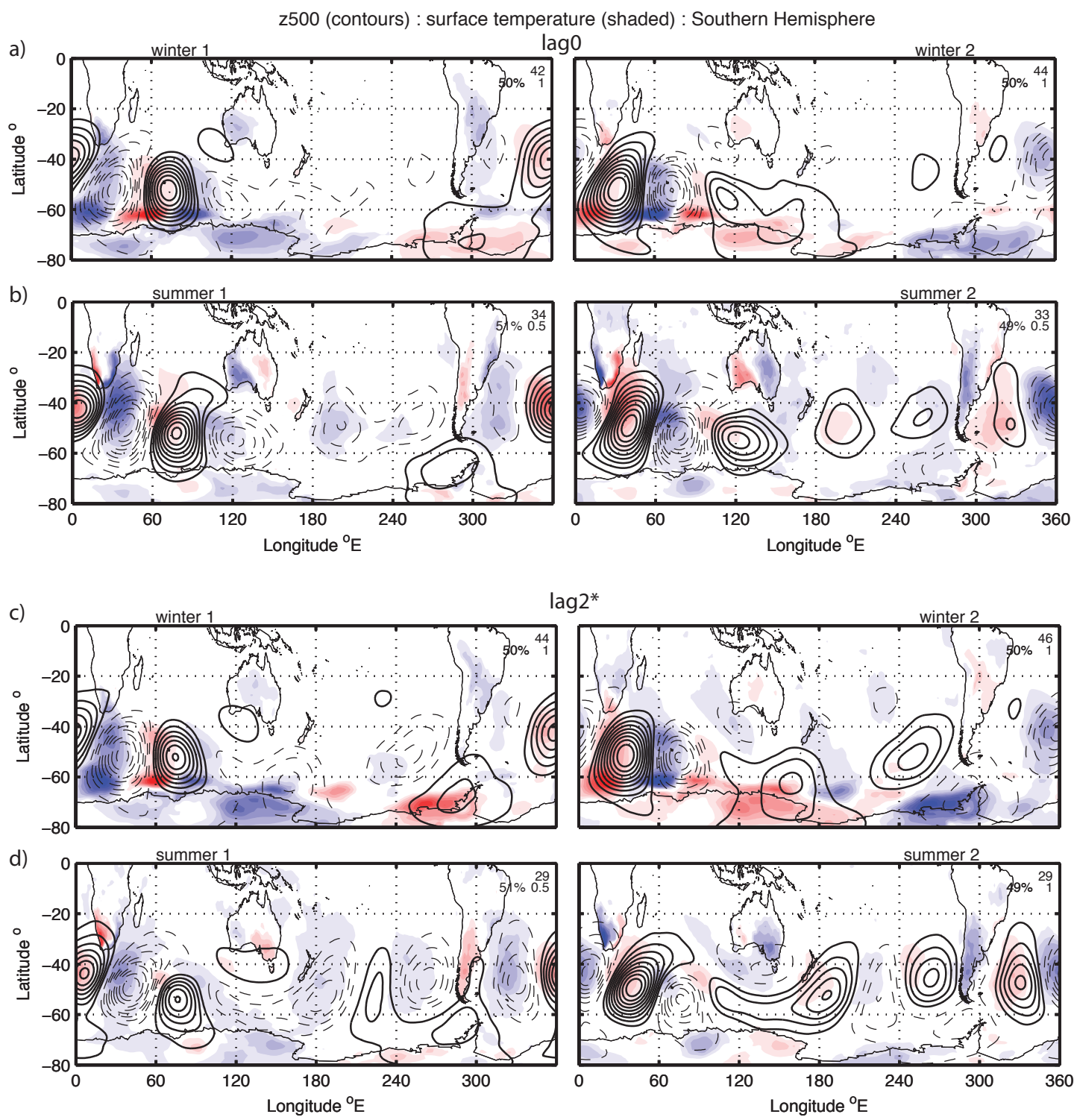

Figure 15: comb-21: Winter and summer $Z_{g 500 h P a}$ anomaly cluster composite states (contours) and $S A T$ anomalies (shaded) for lag 0 (a \& b) and for optimal lag 2 (c \& d).

Markovian effects are incorporated, there is evidence that ill-conditioning acts to degrade the identification of metastable states.

\subsection{Summary of Southern Hemisphere teleconnections:}

- The SAM patterns are present in all models (Bernoulli, Markov and non-Markovian) for the multi-60 experiment. Here, all dimensions communicate with each other, emphasizing the multiscale nature of the SAM and the important role of higher dimensions and their impact on the leading mode of variability. Significantly longer time series than available were found to be required in order to reduce sampling error and the effect of ill-conditioning - necessary conditions that prohibit the investigation of non-Markovian (memory) effects for this case. 
- As with the NH, CWP and blocking modes appear where the dimensionality is reduced to the leading 10-20 PCs, but coupling across dimensions allowed. For the baroclinic - barotropic cases and 10-20 PCs, blocking in the Tasman Sea was found to be most evident for the Markovian case, in part due to the more persistent PSA mode downstream. For the equivalent barotropic multi-60 case the SAM is present at all lags, whereas more than one day of memory is required to isolate the more persistent PSA and Indian Ocean blocking modes for baroclinic-barotropic comb-60 case.

- Diagonalization reveals the CWP with particular regions more or less prevalent as memory is increasingly taken into account. However, the seasonal variation between the winter (subtropical) and summer (polar) jets has the most pronounced impact on the structure of the persistent states, which is the case for both the multi-60-1D or comb-60-1D cases.

- In contrast to the multi-level calculation (SAM), considering the leading 20 independent PCs at each level (comb-60) reveals the CWP (Bernoulli) and PSA1 (Bernoulli-Markov) teleconnection patterns. This indicates the equivalent barotropic nature of the wave-3-SAM patterns and a significant role for baroclinic instability in the CWP, PSA2 teleconnections and for SH blocking.

- For the comb-21 case, i.e. Bernoulli without memory, CWP manifest only in the Indian Ocean sector. For the optimal lag 2 and longer, the PSA2 pattern in the Pacific forms in both seasons, with a clear CWP now present across the full hemisphere in the summer.

- Severe truncation to the leading 10 modes results in a significant degradation of the composite states when memory effects are included in the model.

\section{Conclusions}

Atmospheric teleconnections occur on a continuum of scales, and with varying degrees of dependence on past sequences of events. These facts require rigorous approaches to the identification of the canonical patterns of the persistent quasi-stationary states of the atmosphere and their secular behaviour. In particular some form of prefiltering or dimension reduction is required to render the computational problem tractable. However, as discussed earlier by Lau et al. [25], one must be aware of the consequences of various prefiltering and or dimension reduction strategies be they EOF/PC analysis, averaging or band pass filtering.

Here, we have used a data-driven nonparametric multiscale approximation to nonstationary dynamical processes to undertake an examination of role of memory and dimensionality in the troposphere. We have examined two classes of teleconnection, one associated with zonally-oriented wave trains and the other distinguished by meridional dipolar structures, and considered their baroclinic and barotropic nature.

At the most fundamental level we find the respective SH and NH CWP teleconnections to be the intrinsic or fundamental modes where coherent structures form on particular CWP nodes at given times with varying degrees of intensity and persistence in response to disturbances in the flow and seasonal changes in the jet structure. In general, we find that the more persistent states are equivalent barotropic, such as the PSA1 in the SH, the NAO and PNA, whereas the less persistent states tend to be baroclinic-barotropic, for example the PSA2 and the relatively transient nature of blocking in the SH.

In the atmospheric sciences EOF/PC analysis is a common method for analysing the large scale variability of teleconnection modes based on capturing the relevant dynamics on a slow manifold. This approach is problematic when one considers that much of the large scale atmospheric variability can be described as transitions between quasi-stationary or metastable states, where the transition from one state to another is initiated by the projection of the variability of the fast modes onto the slow manifold. The recognition of the necessity to parameterise the effects of the fast scales has led to application of stochastic modelling approaches to determine reduced order models. In multi-scale systems where there is no clear scale separation, it is not obvious that assumptions of a slow manifold are even valid. As there is no rigorous criteria for determining the potential effects of ill-conditioning for non-stationary timeseries where secular behaviour is present, and given that we cannot a priori discriminate between different models based on dimension alone, systematic sensitivity experiments are required for given particular choices of (reduced) dimension. 
Remarkably, there are few such studies in the literature and it is still commonplace for arbitrary approaches to data reduction for the analysis of climate data and even fewer studies where the unresolved scales are parameterised.

This study has been an attempt to further illustrate some of the dangers of data reduction and to point out the benefits of a systematic approach to analysis of timeseries where secular behaviour and memory effects are present. We find that a careful and systematic dimension reduction strategy can reveal differing aspects of the physical nature of the large scale teleconnections and lead to a clearer recognition of when and where ill-conditioning and overfitting can occur.

Acknowledgement: TJO acknowledges funding support of an Australian Research Council Future Fellow (grant FT120100008). DPM was supprted by the Australian Climate Change Science Program, JSR was supported by the Grains Research Development Corporation, IH was supported by the DFG Mercator Fellowship in SFB 1114 and by the project "Spontaneous Imbalance" in the DFG Forschergruppe MS-GWaves. CLEF was supported by the German Research Foundation (DFG) through the cluster of excellence CliSAP.

\section{References}

[1] Akaike H., Information Theory and an Extension of the Maximum Likelihood Principle, in Selected Papers of Hirotugu Akaike, Eds. E. Parzen, K. Tanabe and G. Kitagawa, Springer, New York (1988).

[2] Ambrizzi T., Hoskins B. J., Hsu H. -H. , Rossby wave propagation and teleconnection patterns in the austral winter, J. Atmos. Sci., 1995, 52(21), 3661-3672

[3] Barnston A. G., Livezey R. E. , Classification, seasonality and persistence of low-frequency atmospheric circulation patterns, Mon. Weather Rev., 1987, 115(6), 1083-1126

[4] Branstator G., Circumglobal teleconnections, the jet stream waveguide, and the North Atlantic Oscillation, J. Clim., 2002, 15(14), 1893-1910

[5] Bouchet F., Simonnet E., Random changes of flow topology in two-dimensional and geophysical turbulence, Phys. Rev. Lett., 2009, 102(9), 094504

[6] Charney J., DeVore J., Multiple flow equilibria in the atmosphere and blocking, J. Atmos. Sci., 1979, 36, 1205-1216

[7] Franzke C. L. E., Horenko I., Majda A. J., Klein R., Systematic Metastable Atmospheric Regime Identification in an AGCM, J. Atmos. Sci., 2009, 66, 1998-2012

[8] Franzke C. L. E., O’Kane T. J., Monselesan D. P., Risbey J. S., Horenko I., Systematic attribution of observed Southern Hemispheric circulation trends to external forcing and internal variability, Nonlin. Processes Geophys. Discuss., 2015, 2 , 675-707

[9] Frederiksen C. S., Zheng X., Grainger S., Simulated modes of inter-decadal predictability in sea surface temperature, Clim. Dyn., 2015, DOI 10.1007/s00382-015-2699-6

[10] Giannakis, D., Majda A. J., Quantifying the Predictive Skill in Long-Range Forecasting. Part I: Coarse-grained predictions in a simple ocean model, J. Climate, 2012 25, 1793-1813

[11] Giannakis, D., Majda A. J., Quantifying the Predictive Skill in Long-Range Forecasting. Part II: Model Error in CoarseGrained Markov Models with Application to Ocean-Circulation Regimes, J. Climate, 2012, 25, 1814-1827

[12] Granger C. W. J., Some recent development in a concept of causality, Econometrics, 1988, 39, 199-211

[13] Horenko I., On Robust Estimation of Low-Frequency Variability Trends in Discrete Markovian Sequences of Atmospheric Circulation Patterns, J. Atmos. Sci., 2009, 66, 2059-2072

[14] Horenko I., On clustering of non-stationary meteorological time series, Dyn. Atmos. Oceans, 2010, 49, 164-187

[15] Horenko I., On the identification of nonstationary factor models and their application to atmospheric data analysis, J. Atmos. Sci., 2010, 67 (5), 1559-1574

[16] Horenko I., Finite Element Approach to Clustering of Multidimensional Time Series, SIAM J. Sci. Comput., 2010, 31(1), 62-83

[17] Horenko I., On the analysis of nonstationary categorical data time series: dynamical dimension reduction, model selection, and applications to computational sociology. Multiscale Model. Simul., 2011, 9(4), 1700-1726

[18] Horenko I., Gerber S., O'Kane T. J., Risbey J. S., Monselesan D. P., On Inference and Validation of Causality Relations in Climate Teleconnections, 2017, Chapt. 5, 136-159, Nonlinear and Stochastic Climate Dynamics, Eds Franzke, C. L. E. and T. J. O'Kane, 483pp, Cambridge University Press, Cambridge UK

[19] Hoskins, B. J. and T. Ambrizzi, Rossby wave propagation on a realistic longitudinally varying flow, J. Atmos. Sci., 1993, $50,1661-1671$ 
[20] Hurrell J. W., Decadal trends in the North Atlantic Oscillation: Regional temperatures and precipitation. Science, 1995, 269, 676-679

[21] Kalnay E., and Coauthors, The NCEP/NCAR 40-Year Reanalysis Project. Bull. Amer. Meteor. Soc., 1996, 77, 437-471 doi:10.1175/1520-0477(1996)077,0437:TNYRP.2.0.C0;2.

[22] Kitsios V., Frederiksen J. S., Zidikheri M. J., Subgrid model with scaling laws for atmospheric simulations J. Atmos. Sci., 2012, 69 1427-1445

[23] Kobayashi, S., Ota Y., Harada Y., Ebita A., Moriya M. , Onoda H., Onogi K., Kamahori H., Kobayashi C., Endo H., Miyaoka K., and Takahashi K., The JRA-55 Reanalysis: General Specifications and Basic Characteristics, J. Meteor. Soc. Japan, 2015, 93(1), 5-48

[24] Kraichnan R. H., Inertial Ranges in Two-Dimensional Turbulence Phys. Fluids, 1967, 10(7), 1417-1423

[25] Lau K.-M., Shey P.-J., Kang I.-S. , Multiscale low-frequency circulation modes in the global atmosphere, J. Atmos. Sci., 1994, 51, 1169-1193

[26] Legras B. and Ghil M., Persistent anomalies, blocking and variations in atmospheric predictability, J. Atmos. Sci., 1985, 42(5), 432-471

[27] Leith C. E., Nonlinear normal mode initialization and quasigeostrophic theory, J. Atmos. Sci., 1980, 37 958-968

[28] Lorenz E., Seasonal and irregular variations of the Northern Hemisphere sea-level pressure profile, J. Meteor., 19518 , 52-59

[29] Lorenz E. N., Attractor sets and quasi-geostrophic equilibrium, J. Atmos. Sci., 1980, 37 1685-1699

[30] Majda A. J., Franzke C. L., Fischer A., Crommelin D. T., Distinct metastable atmospheric regimes despite nearly Gaussian statistics: A paradigm model, Proc. Natl. Acad. Sci. USA, 2006, 103, 8309-8314

[31] Majda, A. J., Gershgorin B., Crommelin D., Normal forms for reduced stochastic climate models. Proc. Natl. Acad. Sci. USA, 2009, 106, 3649-3653

[32] Metzner P., Putzig L., Horenko I., Analysis of persistent nonstationary time series and applications, Comm. Appl. Math. Comp. Sci., 2012, 7(2), 175-229

[33] Mo K. C., Higgins R. W., The Pacific-South American Modes and Tropical Convection during the Southern Hemisphere Winter, J. Atmos. Sci., 1988, 126, 1581-1596

[34] Nadiga B. T., O'Kane T. J., Low-Frequency Regime Transitions and Predictability of Regimes in a Barotropic Model, 2017, Chapt. 5, 136-159, Nonlinear and Stochastic Climate Dynamics, Eds Franzke, C. L. E. and T. J. O’Kane, 483pp, Cambridge University Press, Cambridge UK

[35] O'Kane T. J., Frederiksen J. S., Statistical dynamical subgrid-scale parameterizations for geophysical flows, Phys. Scr., 2008, 132014033

[36] O’Kane T. J., Monselesan D. P., Risbey J. S., A multiscale re-examination of the Pacific South American pattern, Mon. Rev. Rev., 2017, 145(3), 379-402

[37] O’Kane T. J., Risbey J. S., Franzke C. L. E., Horenko I., Monselesan D. P., Changes in the meta-stability of the mid-latitude Southern Hemisphere circulation and the utility of nonstationary cluster analysis and split flow blocking indices as diagnostic tools, J. Atmos. Sci., 2013, 70(3), 824-842

[38] O’Kane T.J., Risbey J. S., Monselesan D. P., Horenko I., Franzke C. L. E., On the dynamics of persistent states and their $\underline{\text { secular trends }}$ in the waveguides of the Southern Heisphere troposphere, Cli. Dynamics, 2015, D0I 10.1007/s00382-0152786-8

[39] Polvani L. M., Waugh D. W., Correa G. J. P., Son S. W., Stratospheric ozone depletion: The main driver of twentieth-century atmospheric circulation changes in the Southern Hemisphere, J. Clim. 2011, 24, 795-812

[40] Phillips N. A. Energy transformations and meridional circulations associated with simple baroclinic waves in a two-level, quasi-geostrophic model, Tellus, 1954, 6(x), 273-286

[41] Risbey J. S., O’Kane T. J., Monselesan D. P., Franzke C. L. E., Horenko I., Metastability of Northern Hemisphere teleconnection modes, J. Atmos. Sci., 2015, 72(1), 35-54

[42] Roscoe H. K., Haigh J. D., Influences of ozone depletion, the solar cycle and the QBO on the Southern Annular Mode Q. J. R. Meteorol. Soc, 2007, 133, 1855-1864

[43] Thompson D. W., and Wallace J. M., The Arctic Oscillation signature in the wintertime geopotential height and temperature fields. Geophys. Res. Lett., 1988, 25, 1297-1300

[44] Thompson, D.W., Wallace J. M., Annular modes in the extratropical circulation. Part I: Month to month variability J. Climate, 2000, textit13, 1000-1016

[45] Stolle, J., Lovejoy S., Schertzer D, The temporal cascade structure of reanalyses and global circulation models $Q$. J. $R$. Meteorol. Soc. 2012, DOI:10.1002/qj.1916

[46] Vallis G., Atmospheric and Oceanic Fluid Dynamics, 2010, 745pp. Cambridge University Press, Cambridge UK

[47] Wallace J. M., Gutzler D. S., Teleconnections in the geopotential height field during the Northern Hemisphere winter. Mon. Wea. Rev., 1981, 109, 784-812

[48] Zidikheri, M. J., Frederiksen, J. S., Stochastic modelling of unresolved eddy fluxes. Geophys. Astrophys. Fluid Dyn., 2010, 104, 323-348. 